\title{
ORIGINAL RESEARCH \\ Fuzzy covering problem of fuzzy graphs and its application to investigate the Indian economy in new normal
}

\author{
Anushree Bhattacharya ${ }^{1} \cdot$ Madhumangal Pal $^{1}$ \\ Received: 15 December 2020 / Revised: 15 March 2021 / Accepted: 25 March 2021 / \\ Published online: 31 March 2021 \\ (c) Korean Society for Informatics and Computational Applied Mathematics 2021
}

\begin{abstract}
In this paper, new concepts of covering of fuzzy graphs are introduced. The definitions of fuzzy covering maps and fuzzy covering graphs of fuzzy graphs are given. Some special types of fuzzy covering graphs are discussed. Some important theorems to find out the fuzzy covering maps as well as fuzzy covering graphs for different types of fuzzy graphs are described. On the basis of the present situation during the current pandemic COVID-19, the world economic status is highly disruptive and deeply affected by the lock-down process. So, this topic is catching the eye for being an application part of this paper. Also, there are globally seventeen goals to sustain our development, which contain the eighth goal 'Decent Work and Economic Growth' having effect on the economy of the world. For this reason, the eighth sustainable development goal is combined with the economic impact of the pandemic for the discussion in the application part. Then some strategies are made to overcome this condition in a better way and perform all the steps to get a better situation in near future.
\end{abstract}

Keywords Fuzzy graph · Fuzzy covering problem · Fuzzy covering graphs · Economic status of India $\cdot$ New normal

Mathematics Subject Classification $05 \mathrm{C} 72$

Financial support of first author offered by DHESTBT (Govt. of West Bengal) Memo No. 353(Sanc.)/ST/P/S\&T/16G-15/2018 is thankfully acknowledged.

Madhumangal Pal

mmpalvu@gmail.com

Anushree Bhattacharya

anumath96@gmail.com

1 Department of Applied Mathematics with Oceanology and Computer Programming, Vidyasagar University, Midnapore 721 102, India 


\section{Introduction}

In 2015, the sustainable development goals (SDGs) are set the global development agenda until 2030. The SDGs are universal plans for all countries to end poverty, protect the planet and ensure prosperity for all. There are 17 goals which include 169 targets. They are intended to be tackled as a group rather than individually, the 17 goals are interlinked. The 17 SDGs are:

(1) No Poverty, (2) Zero Hunger, (3) Good Health and Well Being, (4) Quality Education, (5) Gender Equality, (6) Clean Water and Sanitation, (7) Affordable and Clean Energy, (8) Decent Work and Economic Growth, (9) Industry, Innovation, and Infrastructure, (10) Reduced Inequalities, (11) Sustainable Cities and Communities, (12) Responsible Construction and Production, (13) Climate Action, (14) Life Below Water, (15) Life on Land, (16) Peace, Justice, and Strong Institutions, (17) Partnerships and Goals. These SDGs are discussed in more detail in [36,44].

The Eighth SDG is "Decent Work and Economic Growth", the index-score value of which is connected to the economic status of any country. Many developing countries in the world, perform as much as possible to achieve the target of the Eighth SDG by taking many necessary treads for this purpose. But, in the last few months all the strategies are becoming meaningless and the backbone of the development is broken due to a huge effect of the current pandemic COVID-19.

\subsection{Impact of COVID-19 in the world economy}

COVID-19 has triggered the deepest global recession in decades. The global economic outlook during the COVID-19 pandemic is a changed world. The COVID-19 pandemic has spread with alarming speed, infecting millions and bringing economic activity to a near-standstill as countries imposed tight restrictions on movement to halt the spread of the virus. The baseline forecast envisions a $5.2 \%$ contraction in global GDP in 2020. The pandemic is expected to plunge most countries into recession in 2020, with per capita income contracting in the largest fraction of countries globally since 1870. The pandemic and efforts to contain it have triggered an unprecedented collapse in oil demand and a crash in oil prices. Should COVID-19 outbreaks persist, should restrictions on movement be extended or reintroduced, or should disruptions to economic activity be prolonged, the recession could be deeper. Under this downside scenario, global growth could shrink by almost 8 percent in 2020 . While the ultimate outcome is still uncertain, the pandemic will result in contractions across the vast majority of emerging markets and economies development.

\subsection{Motivation of the work}

To handle real-life all uncertainties, fuzzy mathematics is the best of all fields of mathematics. Specially, the fuzzy graph is one of the strong tools to model the relationship between various features with impreciseness. For the first time, the concept of fuzzy sets is given by Zadeh [50] and fuzzy graphs are introduced by Rosenfield [34]. Yager [49] has described knowledge-based defuzzification. Also, facility location problems 
are very essential for modeling real-life problems. The idea of covering fuzzy graphs will make the work smooth to analyze a network and console the demand of any fuzzy system. As, the economy is the most important part of our life, which has a markable damage during this pandemic and it will deeply affect our daily-life; so this topic is of much importance to analyze and make necessary strategies to overcome this situation. Fuzzy covering map and fuzzy covering graph of a fuzzy graph are totally new concepts in the area of covering concepts of fuzzy graphs.

In this paper, the new definition of fuzzy covering maps and graphs of fuzzy graphs are introduced. To mix the taste of facility location concept in this context for covering maps and graphs of fuzzy graphs, the vertices of a fuzzy graph are considered as facilities as well as demand points depending on the considered situation of the problem.

\subsection{Literature review}

There are many works in the field of covering of fuzzy graphs. In the area of vertex covering, Hastad [17] showed that approximating vertex cover within constant factors less than 7/6 is NP-hard. The factor of Hastad to 1.36 is improved by Dinur and Safra [13]. The randomness in the problem and the probability theory to minimum weight edge covering problem are discussed by $\mathrm{Ni}$ [26]. On the other side, the credibility theory to find the fuzzy minimum weight edge cover in a fuzzy graph was discussed by him in 2008 [27]. The fuzzy graphs have been used by Koczy in evolution and optimization of networks [18]. Pramanik and Pal [30] have introduced a fuzzy $\phi$-tolerance competition graph. Samanta and Pal [42] have proved results on $k$-competition and $p$-competition fuzzy graphs, they also studied with fuzzy tolerance graphs [41] and fuzzy planar graphs [43]. Rashmanlou et al. [33] have studied bipolar fuzzy graphs. Interval-valued fuzzy planar graphs are nicely discussed by Pramanik et al. [29]. In [39], Samanta et al. described new concepts of fuzzy planar graphs. Samanta et al. [38] also worked on some results on $m$-step fuzzy competition graphs. Rashmanlou and Pal [32] have provided notable theories and important results about the isometry on interval-valued fuzzy graphs. Sahoo and Pal [37] have done wonderful study on intuitionistic fuzzy competition graphs. Samanta et al. [40] have developed the methodology of fuzzy colouring of fuzzy graphs. Ghorai and Pal [15] have worked on $m$-polar fuzzy planar graphs, they also have given theories on, "Faces and dual of $m$-polar fuzzy planar graphs" [16]. An unified approach to fuzzy graph problems is given by Blue et al. [9]. Chang and Zadeh [11] have worked on fuzzy mappings. Conditional covering problem is considered by Chaudhry [12]. Wieslaw and Talebi [48] have given idea about operations on level graph of bipolar fuzzy graphs. Cayley fuzzy graph on fuzzy group is discussed by Talebi [45]. Borzooei et al. [10] have worked on regularity of vague graphs. Also, Talebi et al. [46] have introduced some new concepts of $m$-polar interval-valued intuitionistic fuzzy graph.

The definition of neutrosophic graphs was introduced by Akram and Shahzadi [5]. Mahapatra et al. [21] has given applications of edge colouring of fuzzy graphs. Some operations on single-valued NGs are given by Akram and Shahzadi [4]. Mahapatra et al. [20] have given the concept of radio fuzzy graphs. Sitara and Akram [6] have 
shown novel applications in decision-making of neutrosophic graphs. Based on bipolar neutrosophic directed hypergraphs, a new decision-making method is introduced by Akram and Luqman [3]. In addition, Naz and Akram [25] have worked on energy in single-valued neutrosophic graphs. Akram [2] elaborately discussed single-valued neutrosophic graphs. A new way of link prediction in social networks is introduced by Mahapatra et al. [19]. The concept of generalized neutrosophic planar graphs are described by Mahapatra et al. [22]. Link prediction in social networks by neutrosophic graphs are studied by Mahapatra et al. [23]. Bhattacharya and Pal [7] have worked with vertex covering problems of fuzzy graphs and given application in CCTV installation. Also, clique covering problems are used in optimization by Bhattacharya and Pal [8]. Pal et al. [28] have discussed modern trends in fuzzy graphs and their applicability. Dubois and Prade [14] have discussed about different operations of fuzzy numbers in their studies.

\subsection{Novelties}

In literature, there are several publications on covering of crisp graphs, but there is a huge scope to work with covering of fuzzy graphs. Also, as per best of our knowledge, there is no work in fuzzy covering maps and graphs of fuzzy graphs. In this paper, some important definitions, works and their applications are provided. The summarized form of the work done are as follows:

(i) Defining fuzzy covering map and fuzzy covering graph of a fuzzy graph.

(ii) Grading the vertices of fuzzy graphs with respect to the vertex membership function.

(iii) Fuzzy covering map and covering graph for different fuzzy graphs like picture fuzzy graphs, fuzzy perfect graphs and $m$-polar fuzzy graphs are emphasised.

Some useful notations and abbreviations are given in the Table 1.

\subsection{Organization of the paper}

The paper is organised as given. In Sect. 2, some basic and important definitions are provided. The problem under consideration is described in Sect. 3. An algorithm to find a fuzzy covering graph of a fuzzy graph is described in Sect. 4. In Sect. 5, some important theorems are given for different fuzzy graphs. The fuzzy covering maps and fuzzy covering graphs for picture fuzzy graphs and neutrosophic fuzzy graphs in Sects. 6 and 7 respectively. In Sect. 8, an application on the impact of COVID-19 in the economy of India with detailed analysis is provided. An entire conclusion is given in Sect. 9.

\section{Preliminaries}

Definition 1 [24] A fuzzy graph $G=(V, \sigma, \mu)$ with the underlying crisp graph $G^{*}=$ $(V, E)$ is a graph defined by the couple of fuzzy membership functions, $\sigma: V \rightarrow$ 
Table 1 Some important notations and abbreviations

\begin{tabular}{ll}
\hline Symbols/notations & Meaning \\
\hline SDG & Sustainable development goals \\
GDP & Gross domestic product \\
GST & Goods and services tax \\
$G=(V, \sigma, \mu)$ & Fuzzy graph \\
$G^{*}=(V, E)$ & Crisp graph \\
$N(u)$ & Neighbourhood of a vertex $u$ \\
$N[u]$ & Closed neighbourhood of a vertex $u$ \\
$\gamma_{N}(G)$ & Minimum neighbourhood value of $G$ \\
$\Gamma_{N}(G)$ & Maximum neighbourhood value of $G$ \\
$G_{I}$ & Index of a fuzzy graph $G$ \\
$C_{I}(G)$ & Covering index of fuzzy graph $G$ \\
$d_{T}(u)$ & Total degree of a vertex $u$
\end{tabular}

$[0,1], \mu: V \times V \rightarrow[0,1]$ where for all $u, v \in V$, every edges in $G=(V, \sigma, \mu)$ fulfils the condition, $\mu(u, v) \leq \sigma(u) \wedge \sigma(v)$.

The idea of covering in fuzzy graphs was presented by Somasundaram. The author also defined node covering and arc covering in fuzzy graphs using effective arcs and scalar cardinality.

Definition 2 [47] The neighbourhood of a vertex $u$ of a fuzzy graph $G=(V, \sigma, \mu)$ is defined by the set,

$$
N(u)=\{v \in V: \mu(u, v) \leq \sigma(u) \wedge \sigma(v)\} .
$$

and, $N[u]=\{u\} \cup N(u)$ is called the closed neighbourhood of $u$.

Definition 3 [47] The term $\delta_{N}(u)=\sum_{v \in N(u)} \sigma(v)$ is called the neighbourhood value of a vertex $u$ for a fuzzy graph $G=(V, \sigma, \mu)$.

Definition 4 The minimum neighbourhood value of $G=(V, \sigma, \mu)$ is,

$$
\gamma_{N}(G)=\min \left\{\delta_{N}(v): v \in V\right\}
$$

and the maximum neighbourhood value of $G=(V, \sigma, \mu)$ is,

$$
\Gamma_{N}(G)=\max \left\{\delta_{N}(v): v \in V\right\} .
$$

Definition 5 [35] Let, $G=\left(V_{1}, E_{1}\right)$ and $C=\left(V_{2}, E_{2}\right)$ be two fuzzy graphs and let $f: V_{2} \rightarrow V_{1}$ be a surjection. Then $f$ is a covering map from $C$ to $G$ if for each $v \in V_{2}$, the restriction of $f$ to the neighbourhood of $v$ is a bijection onto the neighbourhood of $f(v)$ in $G$.

If there exists a covering map from $C$ to $G$, then $C$ is a covering graph, or a lift of G. 


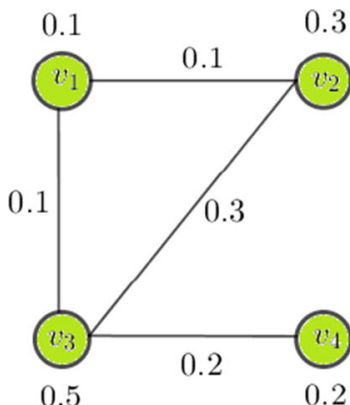

$G$

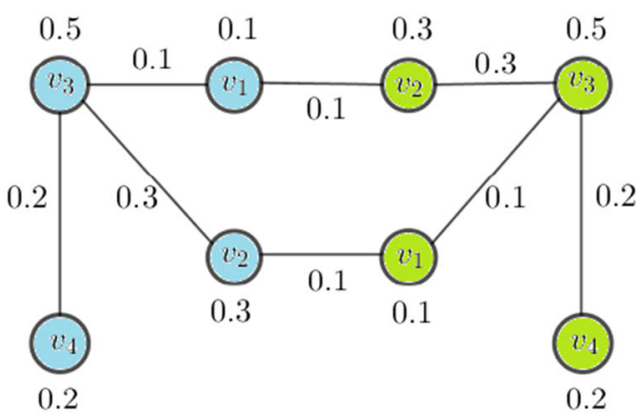

$H$

Fig. 1 Covering graph of a fuzzy graph by crisp covering map

Example 1 We consider a fuzzy graph and using the crisp covering map, we find out the crisp covering graph of the fuzzy graph shown in the Fig. 1.

In the Fig. $1, H$ is the covering map of the fuzzy graph $G$ by considering a crisp covering map from the vertex set of $H$ to the vertex set of $G$. In Fig. 1, the greencoloured vertices of the graph $G$ are considered as facility nodes. Here, the covering radius of $G$ is considered as 2 . That is, we can repeat the facility nodes which are situated within 2-edge distances from any vertex present in the fuzzy graph. Also, the edge-membership values remain the same as in the original fuzzy graph by considering the crisp covering map between the vertex sets of $G$ and $H$. In $H$, the repeated facility nodes are the sky-coloured vertices.

Definition 6 The equality function on $(V \times V)$ for a fuzzy graph $G=(V, \sigma, \mu)$ to define the fuzzy covering map is denoted by $E: V \times V \rightarrow[0,1]$ and given by,

$$
E(u, v)=\frac{\min \{\sigma(u), \sigma(v)\}}{\max \{\sigma(u), \sigma(v)\}} .
$$

Also, $E(u, v) \leq 1$ implies $\sigma(u) \leq \sigma(v)$ for $u, v \in V$.

Definition 7 Let, $G_{1}=\left(V_{1}, \sigma_{1}, \mu_{1}\right)$ and $G_{2}=\left(V_{2}, \sigma_{2}, \mu_{2}\right)$ be two connected fuzzy graphs.

For two vertex sets $V_{1}$ and $V_{2}$, let $\tilde{f}: V_{2} \rightarrow V_{1}$ be a fuzzy covering map of $G_{1}$ on $G_{2}$ with respect to a equality function $E$ on both $V_{1}$ and $V_{2}$, then

(i) $\chi_{\tilde{f}}$ is surjective fuzzy function i.e., $\forall v \in V_{2}$, there exists $u \in V_{1}$ such that $\chi_{\tilde{f}}(u, v)>0$.

(ii) $\chi_{\tilde{f}}$ is injective fuzzy function i.e.,

$$
\operatorname{chi} i_{\tilde{f}}(u, z) \wedge \chi_{\tilde{f}}(v, w) \wedge E(z, w) \leq E(u, v),
$$

$\forall u, v \in V_{1}$ and $\forall z, w \in V_{2} ;$

where, $\chi_{\tilde{f}}$ is the membership function of $\tilde{f}$.

If the above hold, $G_{1}=\left(V_{1}, \sigma_{1}, \mu_{1}\right)$ is called a fuzzy covering graph of $G_{2}=$ $\left(V_{2}, \sigma_{2}, \mu_{2}\right)$. 

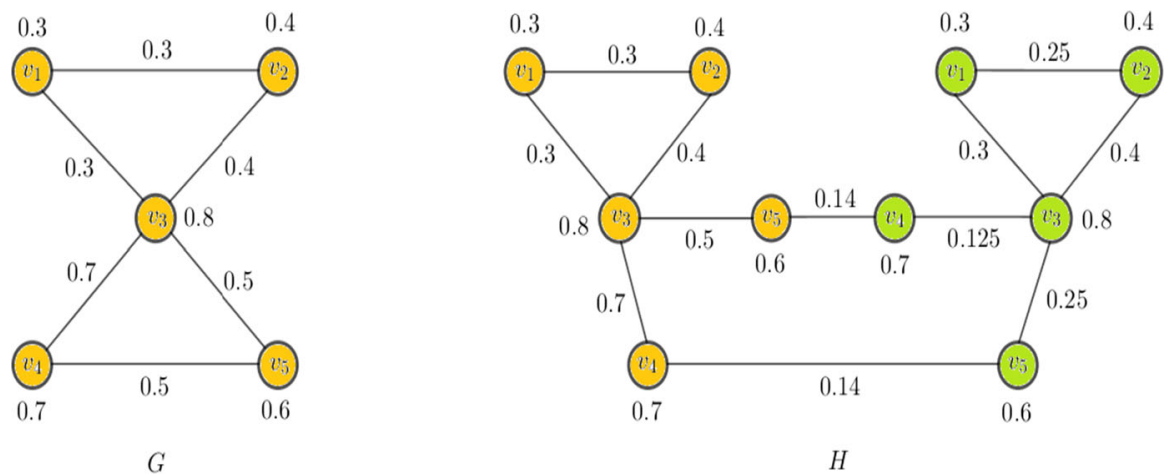

Fig. 2 Fuzzy covering graph of a fuzzy graph

Note 1 Clearly, a fuzzy graph $G=(V, \sigma, \mu)$ be a fuzzy covering graph of itself. In this case, this fuzzy graph is called a fuzzy self-covering graph.

Also, the fuzzy covering map and fuzzy covering graph for a fuzzy graph is not unique.

Example 2 Let us consider a fuzzy graph and using the definition of fuzzy covering map, we have to find out the fuzzy covering graph of the considered fuzzy graph. The considered graph is named as $G$, whereas the fuzzy covering graph is denoted by $H$. Now, we add the facility vertices of the given graph by the help of a fuzzy covering map to construct the graph $H$. Here, the neighbouring properties of the vertices of the fuzzy graph are conserved and the covering map satisfied all the properties of bijective fuzzy function. In $H$, we repeat all the facilities of $G$ to cover the fuzzy graph $G$ by splitting the vertices at $v_{4}$ and $v_{5}$. As shown in Fig. 2 , the facility vertex $v_{4}$ is adjacent vertex of the vertices $v_{5}$ and $v_{1}$; also the facility vertex $v_{5}$ is adjacent to the vertices $v_{4}$ and $v_{1}$. This neighbourhood property of the vertices $v_{4}$ and $v_{5}$ are kept in the fuzzy covering map $H$ of fuzzy graph $G$.

In the Fig. 2, the fuzzy graph $H$ is a fuzzy covering map of the fuzzy graph $G$ by considering the fuzzy covering map $\tilde{f}$ which have the membership function, $\chi_{\tilde{f}}$ is given by

$\chi_{\tilde{f}}(u, v)=\{\sigma(u) \wedge \sigma(v)\} \min \left\{\left\{E\left(u, u_{i}\right): u_{i} \in N(u)\right\} \wedge\left\{E\left(v, v_{i}\right): v_{i} \in N(v)\right\}\right\}$.

The above defined membership function is a fuzzy surjective as well as fuzzy injective function, therefore $H$ is a fuzzy covering graph of the given fuzzy graph $G$.

In $H$, yellow-coloured vertices are the same as in the original graph $G$ and the green-coloured vertices are the repeated facility nodes to construct the fuzzy covering graph $H$ of the fuzzy graph $G$.

Definition 8 Let, $G_{1}=\left(V_{1}, \sigma_{1}, \mu_{1}\right)$ and $G_{2}=\left(V_{2}, \sigma_{2}, \mu_{2}\right)$ be two fuzzy graphs.

For two vertex sets $V_{1}$ and $V_{2}$, let $\tilde{f}: V_{2} \rightarrow V_{1}$ be a strong fuzzy covering map of $G_{1}$ on $G_{2}$ with respect to a equality function $E$ on both $V_{1}$ and $V_{2}$, then 
(i) $\chi_{\tilde{f}}$ is strong surjective fuzzy function i.e., $\forall v \in V_{2}$, there exists $u \in V_{1}$ such that

$$
\chi_{\tilde{f}}(u, v)=1 .
$$

(ii) $\chi_{\tilde{f}}$ is injective fuzzy function i.e.,

$$
\chi_{\tilde{f}}(u, z) \wedge \chi_{\tilde{f}}(v, w) \wedge E(z, w) \leq E(u, v),
$$

$\forall u, v \in V_{1}$ and $\forall z, w \in V_{2}$.

If the above hold, $G_{1}=\left(V_{1}, \sigma_{1}, \mu_{1}\right)$ is called a strong fuzzy covering graph of $G_{2}=\left(V_{2}, \sigma_{2}, \mu_{2}\right)$.

Definition 9 [31] Total degree of a vertex $u$ of a fuzzy graph $G=(V, \sigma, \mu)$ is defined as,

$$
d_{T}(u)=\sum_{u_{i} \in N(u)} \mu\left(u, u_{i}\right)+\sigma(u) .
$$

Definition 10 Index of a fuzzy graph $G=(V, \sigma, \mu)$ is defined as,

$$
G_{I}=\sum_{u \in G} d_{T}(u)
$$

And, the term 'Covering index' is used for the index of the covering graph of a fuzzy graph and it is denoted by $C_{I}(G)$.

Note 2 (i) As finite numbers of vertices present in the vertex-sets, so the number of fuzzy covering maps from one fuzzy graph to another fuzzy graph is finite always and countable.

(ii) Fuzzy covering map is clearly a fuzzy isomorphism. That is, $G_{1}$ is homeomorphic to $G_{2}$.

(iii) Also, a fuzzy covering map is a fuzzy homomorphism.

Definition 11 For any graph $G$, it is possible to construct the bipartite double cover of $G$, which is a bipartite graph and a double cover of $G$. The bipartite double cover of $G$ is the tensor product of graphs $G \times K_{2}$ (Fig. 3).

If $G$ is already bipartite, its bipartite double cover consists of two disjoint copies of $G$. A graph may have many different double covers other than the bipartite double cover.

Definition 12 The vertices of a fuzzy graph $G=(V, \sigma, \mu)$ are graded with respect to the vertex membership function as follows:

(i) $\sigma(u)=1$ implies, $u$ is called the strongest vertex, for $u \in V$.

(ii) $\sigma(u) \in(0.7,1)$ implies, $u$ is called first grade vertex, for $u \in V$.

(iii) $\sigma(u) \in(0.5,0.7)$ implies, $u$ is called second grade vertex, for $u \in V$.

(iv) $\sigma(u) \in(0,0.5)$ implies, $u$ is called third grade vertex, for $u \in V$. 


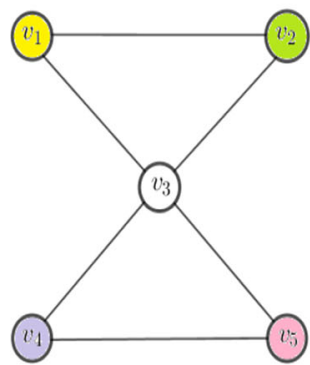

G

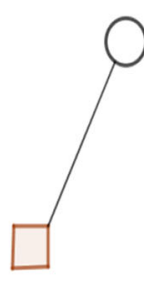

$K_{2}$

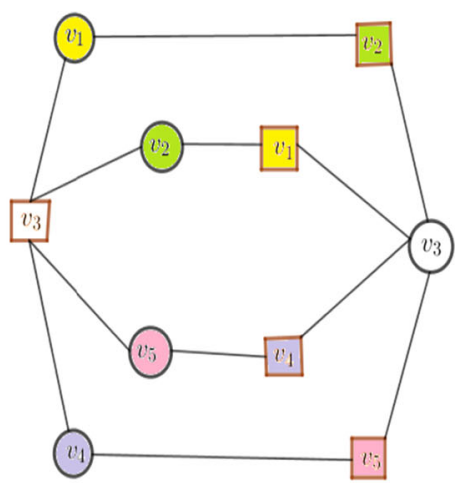

H

Fig. 3 Double covering of a fuzzy graph

Definition 13 [47] Let, $G$ be the composition $G_{1}\left[G_{2}\right]$ of graph $G_{1}$ with $G_{2}$. Let $\left(\sigma_{i}, \mu_{i}\right)$ be a fuzzy subgraph of $G_{i}$, for $i=1,2$. Then $\left(\sigma_{1} \circ \sigma_{2}, \mu_{1} \circ \mu_{2}\right)$ is a fuzzy subgraph of $G_{1}\left[G_{2}\right]$, where

$$
\left(\mu_{1} \circ \mu_{2}\right)\left(\left(u_{1}, u_{2}\right)\left(v_{1}, v_{2}\right)\right)=\min \left\{\left(\sigma_{1} \circ \sigma_{2}\right)\left(\left(u_{1}, u_{2}\right)\right),\left(\sigma_{1} \circ \sigma_{2}\right)\left(\left(v_{1}, v_{2}\right)\right)\right\} .
$$

The fuzzy graph $\left(\sigma_{1} \circ \sigma_{2}, \mu_{1} \circ \mu_{2}\right)$ is called the composition of $\left(\sigma_{1}, \mu_{1}\right)$ with $\left(\sigma_{2}, \mu_{2}\right)$.

Definition 14 [1] Let $G^{*}=(V, E)$ be a graph. A pair $G=(A, B)$ is called a picture fuzzy graph on $G^{*}$ where $A=\left(\mu_{A}, \eta_{A}, v_{A}\right)$ is a picture fuzzy set on $V$ and $B=$ $\left(\mu_{B}, \eta_{B}, v_{B}\right)$ is a picture fuzzy set on $E \subset V \times V$ such that for each $\operatorname{arc} u v \in E$,

$$
\begin{aligned}
& \mu_{B}(u, v) \leq \min \left(\mu_{A}(u), \mu_{A}(v)\right), \eta_{B}(u, v) \leq \min \left(\eta_{A}(u), \eta_{A}(v)\right), v_{B}(u, v) \\
& \quad \geq \max \left(v_{A}(u), v_{A}(v)\right) .
\end{aligned}
$$

Definition 15 [15] An $m$-polar fuzzy graph is a pair $G=(C, D)$, where $C: V \rightarrow$ $[0,1]^{m}$ is an $m$-polar fuzzy set in $V$ and $D: V \times V \rightarrow[0,1]^{m}$ is an $m$-polar fuzzy relation on $V$ such that $p_{i} \circ D(x y) \leq \inf \left\{p_{i} \circ C(x), p_{i} \circ C(y)\right\}$ for all $x, y \in V$.

Definition 16 A fuzzy graph $G=(V, \sigma, \mu)$ on $(V, E)$ is called a perfect fuzzy graph if $\sigma(v)=1$, for all $v \in V$.

\section{Problem under consideration}

Consider a connected, undirected, unweighted fuzzy graph $G=(V, \sigma, \mu)$ with the following facility location problem as a covering problem of the fuzzy graph.

Let, the fuzzy graph $G=(V, \sigma, \mu)$ be given and some facilities $(S(R))$ are already located with covering radius say $R$. Now, introduced some more facilities $\left(S\left(R^{\prime}\right)\right)$ in $G$ with given covering radius $R\left(\leq R^{\prime}\right)$, such that the cost of $\left(S(R) \cup S\left(R^{\prime}\right)\right)$ is 
maximum. In this paper, we consider the covering radius as a length in a fuzzy graph in terms of the number of edges between two vertices of that fuzzy system.

This problem can be referred to as upgradation of the network.

The above problem can be solved by the flavour of fuzzy covering map and fuzzy covering graph of a fuzzy graph. Since, fuzzy covering map is an edge-preserving bijection from one fuzzy graph to another fuzzy graph; therefore, the given fuzzy system will be covered by adding some existing facilities which are more relevant for maximum coverage and maximum cost of the fuzzy network, also they are situated within a certain covering radius of the fuzzy graph. This locating process of the facilities in the fuzzy graph is determined by the definition of fuzzy covering map.

In this covering concept, we consider the cost of covering for a fuzzy covering graph of a fuzzy graph as the covering index of that fuzzy graph throughout the paper. The main aim is to maximize the cost of covering by repeating a minimum number of facilities.

\section{Algorithm to construct fuzzy covering graph of a fuzzy graph}

\section{Algorithm A1}

Input An undirected, connected, unweighted fuzzy graph $G=\left(V_{1}, \sigma_{1}, \mu_{1}\right)$ with a given covering radius considering the vertices as facility nodes and the equality function $E$ for the vertex set.

Output Fuzzy covering graph $H=\left(V_{2}, \sigma_{2}, \mu_{2}\right)$ of the fuzzy graph $G$.

Step-1 Find a fuzzy function $\tilde{f}: V_{2} \rightarrow V_{1}$ with respect to the equality function $E$; and $\chi_{\tilde{f}}$ is the membership function of $\tilde{f}$.

Step-2 Determine the set $A=\left\{u \in V_{1}: u\right.$ repeat in $\left.V_{2}\right\}$, for upgradation of the network.

Step-3 If $u_{i} \in N(u)$, then find $E\left(u, u_{i}\right)$ for $u \in A$.

if $v_{i} \in N(v)$, then find $E\left(v, v_{i}\right)$ for $v \in A$.

compute, $\min \left\{E\left(u, u_{i}\right): u_{i} \in N(u)\right\}$ and $\min \left\{E\left(v, v_{i}\right): v_{i} \in N(v)\right\}$.

Step-4 Find,

$\chi_{\tilde{f}}(u, v)=\left\{\sigma_{1}(u) \wedge \sigma_{1}(v)\right\} \min \left\{\left\{E\left(u, u_{i}\right): u_{i} \in N(u)\right\} \wedge\left\{E\left(v, v_{i}\right): v_{i} \in N(v)\right\}\right\}$,

for $u, v \in V_{1}$.

Step-5 If $\forall v \in V_{2}$, there exists $u \in V_{1}$ such that $\chi_{\tilde{f}}(u, v)>0$

then

if

$$
\chi_{\tilde{f}}(u, z) \wedge \chi_{\tilde{f}}(v, w) \wedge E(z, w) \leq E(u, v) ; \forall u, v \in V_{1}, \forall z, w \in V_{2}
$$

then go to Step-6

otherwise, go to Step-1. 
Step-6 Determine,

$$
\mu_{2}(x, y)= \begin{cases}\sigma_{1}(x) \wedge \sigma_{1}(y) \wedge E(x, y) & \text { if } \quad E(x, y) \in(0,0.5) \\ \sigma_{1}(x) \wedge \sigma_{1}(y) \wedge\{1-E(x, y)\} & \text { if } \quad E(x, y) \in(0.5,1]\end{cases}
$$

for $x, y \in V_{2}$.

Step-7 $\sigma_{2}(u)=\sigma_{1}(x)$ for all $x \in V_{1}$, for all $u \in V_{2}$.

Step-8 Construct the fuzzy graph $H=\left(V_{2}, \sigma_{2}, \mu_{2}\right)$, where $\tilde{f}$ is the fuzzy covering map.

Step-9 Stop.

\section{Some theorems}

In this portion, some important theorems on fuzzy covering maps and fuzzy covering graphs are described for different types of fuzzy graphs. Also, the theorems are discussed by considering some relevant examples.

Theorem 1 Let, $G_{1}=\left(V_{1}, \sigma_{1}, \mu_{1}\right)$ and $G_{2}=\left(V_{2}, \sigma_{2}, \mu_{2}\right)$ be two fuzzy graphs. If $G_{1} \circ G_{2}$ is the composition graph of the two fuzzy graphs $G_{1}$ and $G_{2}$, then $C_{I}\left(G_{1}\right)+C_{I}\left(G_{2}\right) \leq C_{I}\left(G_{1} \circ G_{2}\right)$. In the other words, the fuzzy covering graph of the composition graph of two fuzzy graphs has more coverage than the total coverage by the fuzzy covering graphs of two individual fuzzy graphs.

Example 3 Now, consider two fuzzy graphs $G_{1}$ and $G_{2}$ given in the Fig. 4 . By the definition of fuzzy covering map and fuzzy covering graph of fuzzy graphs, clearly any fuzzy graph can be a fuzzy covering graph of it's own. Therefore, $H_{1}$ and $H_{2}$ are the fuzzy covering graphs of the fuzzy graphs $G_{1}$ and $G_{2}$ respectively, where $H_{1}$ has the same structure and behaviour like $G_{1}$ and $H_{2}$ has the said same as $G_{2}$. The fuzzy covering graphs are also shown in Fig. 4.

Therefore, the covering index of the fuzzy graph $G_{1}$ is given by:

$$
C_{I}\left(G_{1}\right)=(0.2+0.3)+(0.2+0.2+0.2)+(0.2+0.4)=1.7
$$

and, the covering index of the fuzzy graph $G_{2}$ is given by:

$$
C_{I}\left(G_{2}\right)=(0.3+0.3)+(0.3+0.5)=1.4
$$

The composition graph of the two fuzzy graphs $G_{1}$ and $G_{2}$ is determined by the help of the definition composition of two fuzzy graphs given in the preliminaries section. The composition graph $G_{1} \circ G_{2}$ is shown in the Fig. 5. The facility vertices of the graph $G_{1} \circ G_{2}$ are violet coloured nodes.

Here, we consider the trivial case of fuzzy covering graphs of $G_{1}$ and $G_{2}$ i.e., the self-covering graphs shown as in $H_{1}$ and $H_{2}$ respectively. In $H_{1}$, all the yellow-coloured vertices remain the same as facility nodes to cover $G_{1}$ and in $H_{2}$, the sky-coloured vertices of $G_{2}$ remain the same as facilities to cover $G_{2}$. 

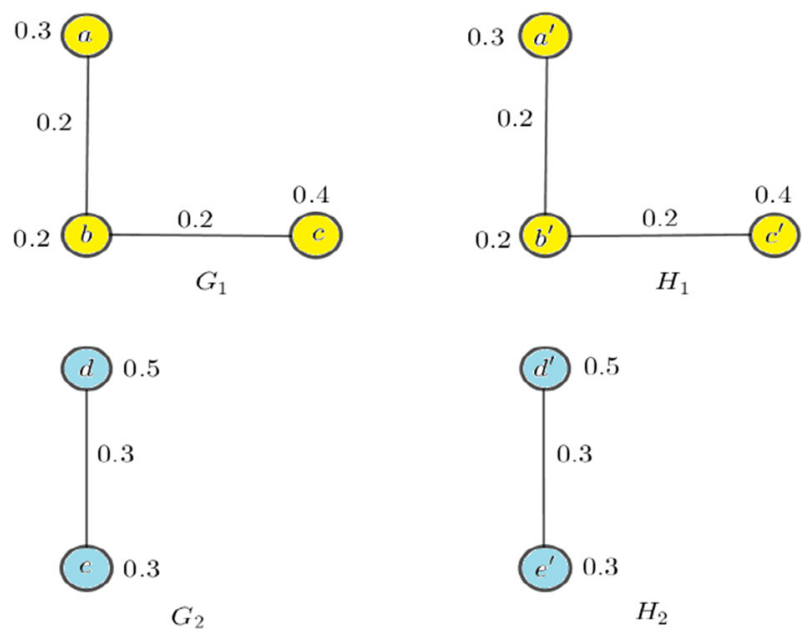

Fig. 4 Fuzzy covering graphs of two fuzzy graphs

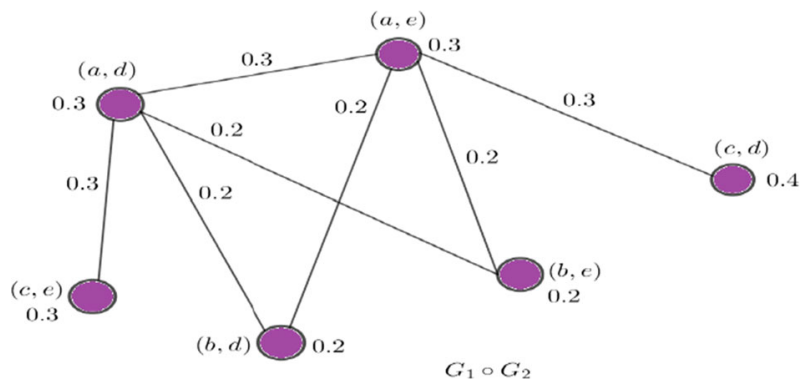

Fig. 5 Composition of given two fuzzy graphs

Now, we have to find out the fuzzy covering graph of the composition graph $G_{1} \circ G_{2}$. For this purpose, consider the fuzzy covering map $\tilde{f}$ where the membership function of $\tilde{f}$ is, $\chi_{\tilde{f}}(u, v)=\{\sigma(u) \wedge \sigma(v)\} \min \left\{\left\{E\left(u, u_{i}\right): u_{i} \in N(u)\right\} \wedge\left\{E\left(v, v_{i}\right): v_{i} \in\right.\right.$ $N(v)\}\}$. In the fuzzy covering map, there are more facilities than the given fuzzy graph to cover up the fuzzy system; then there will also be more edges. For this purpose, we assume that the vertex membership values for the facility nodes remain unchanged. The edge-membership function is redefined for those new edges with the help of the equality function as follows:

$$
\mu(x, y)=\left\{\begin{array}{lll}
\sigma(x) \wedge \sigma(y) \wedge E(x, y) & \text { if } & E(x, y) \in(0,0.5) \\
\sigma(x) \wedge \sigma(y) \wedge\{1-E(x, y)\} & \text { if } \quad E(x, y) \in(0.5,1]
\end{array}\right.
$$

By the above considerations, we get the fuzzy covering graph of the composition graph of the given two fuzzy graphs is given in the Fig. 6. In $G$, the yellow-coloured vertices are the repeated facilities of $G_{1} \circ G_{2}$ to get maximum coverage with maximum covering index. 


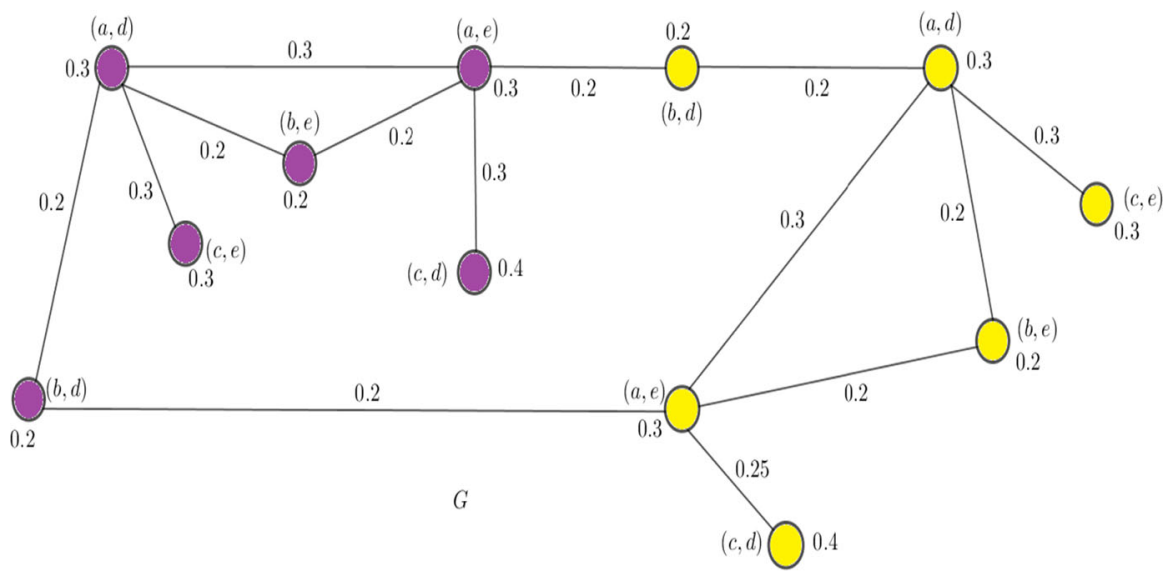

Fig. 6 Fuzzy covering graph of the composition graph

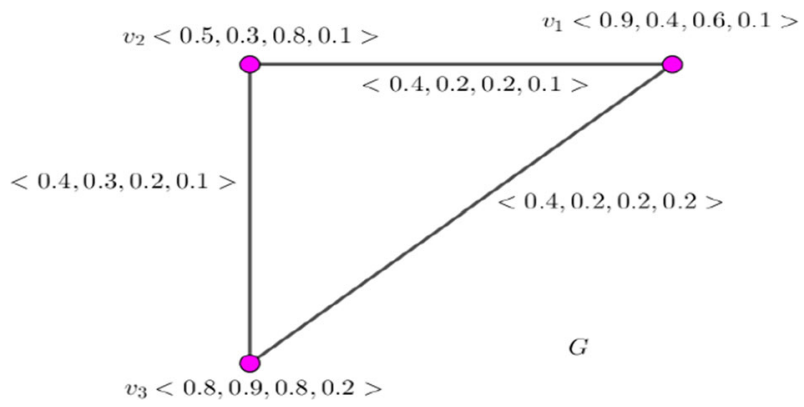

Fig. 7 Considered $m$-polar fuzzy graph

Now, the covering index of $\left(G_{1} \circ G_{2}\right)=$ Index of $G=(0.3+1)+(0.3+0.95)+$ $(0.2+0.4)+(0.3+0.3)+(0.2+0.4)+(0.4+0.25)+(0.2+0.4)+(0.3+0.95)+$ $(0.4+0.25)+(0.3+1)+(0.2+0.4)+(0.3+0.3)=10$.

Therefore, $C_{I}\left(G_{1}\right)+C_{I}\left(G_{2}\right) \leq C_{I}\left(G_{1} \circ G_{2}\right)$ (proved).

Theorem 2 Let us consider an m-polar fuzzy graph, a pair $G=(C, D)$, where $C$ : $V \rightarrow[0,1]^{m}$ is an m-polar fuzzy set in $V$ and $D: V \times V \rightarrow[0,1]^{m}$ is an $m$ polar fuzzy relation on $V$ such that $p_{i} \circ D(x y) \leq \inf \left\{p_{i} \circ C(x), p_{i} \circ C(y)\right\}$ for all $x, y \in V$. Then there is a fuzzy covering map whose membership function is given by: $\chi_{\tilde{f}}(u, v)=\min \left\{p_{i} \circ C(u), p_{i} \circ C(v)\right\}$ for $u, v \in V$. That is, a m-polar fuzzy covering graph of a m-polar fuzzy graph by considering some additional vertices which are the facility nodes of the fuzzy graph.

Example 4 Let us consider a $m$-polar fuzzy graph $G$ given by the Fig. 7. As in the statement of the above theorem, considering the same fuzzy covering map together with it's membership function; we can find out an $m$-polar fuzzy graph as a fuzzy covering graph of the fuzzy graph $G$.

By adding some more existing facilities of $G$ in a new fuzzy graph $H$, we can determine the $m$-polar fuzzy covering graph. For this purpose, we define the edge- 


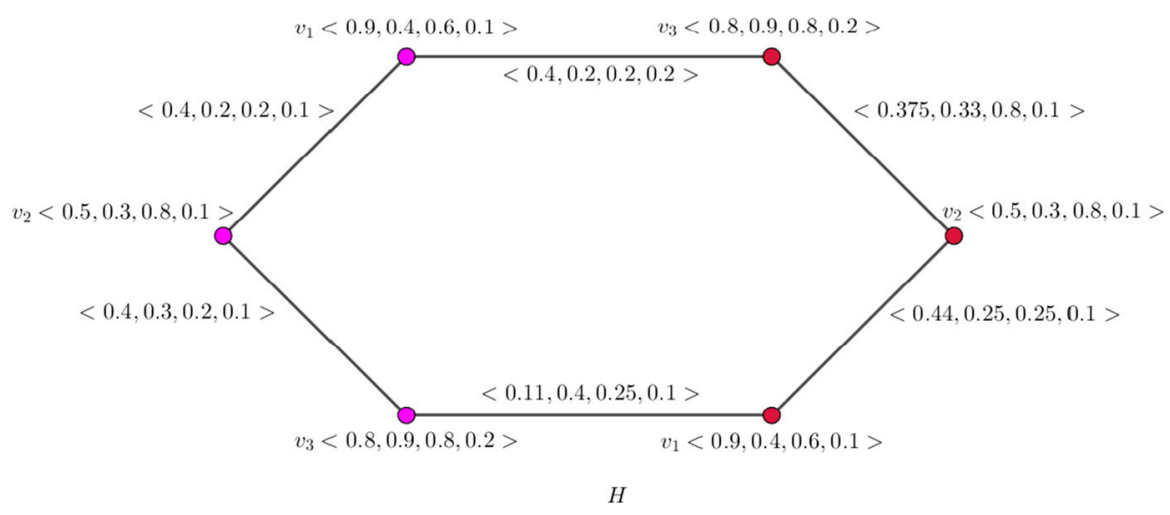

Fig. 8 Fuzzy covering graph of the $m$-polar fuzzy graph

membership function to evaluate the edge-membership values of the new edges in the fuzzy covering graph as follows:

$$
\mu(x, y)= \begin{cases}C(x) \wedge C(y) \wedge E(x, y) & \text { if } \quad E(x, y) \in(0,0.5) \\ C(x) \wedge C(y) \wedge\{1-E(x, y)\} & \text { if } \quad E(x, y) \in(0.5,1]\end{cases}
$$

In Fig. 10, the pink-coloured vertices are the facilities of the fuzzy graph $G$ and let, the covering radius is 2 . The fuzzy covering graph $H$ of the fuzzy graph $G$ is obtained by repeating the facilities $v_{1}, v_{2}, v_{3}$ which are red-coloured in $H$ and the new edges have their membership values determined by the help of the definition of fuzzy covering map and equality function. Then, using the considerations, we have the fuzzy covering graph $H$ which is also an $m$-polar fuzzy graph is given in the Fig. 8.

Theorem 3 The fuzzy covering graph of a fuzzy perfect graph is always a crisp graph and the fuzzy covering map is always a strong fuzzy covering map for a fuzzy perfect graph.

Let, $G=(V, \sigma, \mu)$ on $(V, E)$ be a perfect fuzzy graph if $\sigma(v)=1$, for all $v \in V$. Consider a fuzzy covering map $\tilde{f}$ whose membership function $\chi_{\tilde{f}}$ is given by $\chi_{\tilde{f}}(u, v)=\{\sigma(u) \wedge \sigma(v)\} \min \left\{\left\{E\left(u, u_{i}\right): u_{i} \in N(u)\right\} \wedge\left\{E\left(v, v_{i}\right): v_{i} \in N(v)\right\}\right\}$. Then, $\chi_{\tilde{f}}(u, v)=1 \forall u, v \in V$. We consider this membership function as the edgemembership function of the new additional edges in the fuzzy covering graph of $G$.

Example 5 Let us consider a fuzzy perfect graph $G$ given in the Fig. 9. As in the statement of the above theorem, we consider the same fuzzy covering map with membership function and same edge-membership function, the fuzzy covering graph $H$ is obtained. This graph $H$ is obviously a crisp graph given in the Fig. 9. Clearly, there exists a fuzzy bijective function between the vertex sets of $G$ and $H$; here the corresponding vertices of $G$ and $H$ are same coloured which are evaluated by the help of the fuzzy covering map. 


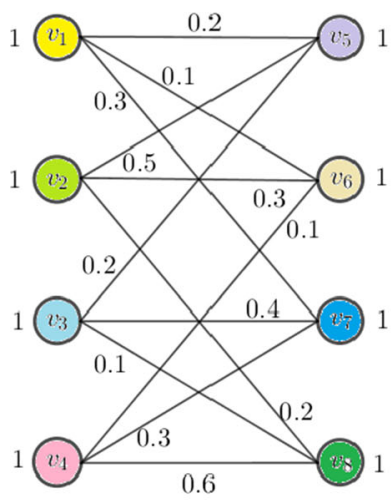

$G$

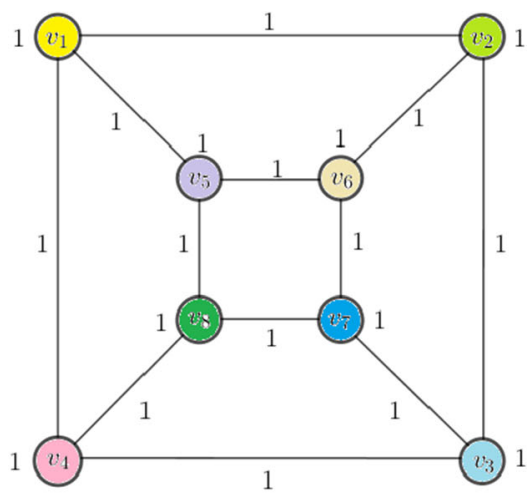

$H$

Fig. 9 Fuzzy covering graph of a perfect fuzzy graph

\section{Fuzzy covering maps for picture fuzzy graph}

The picture fuzzy graph is a particular type of fuzzy graph which includes more conditions for its vertex and edge membership functions. Picture fuzzy graphs are more relevant to use in any real-life fuzzy environment for mathematical modelling in graph theoretic approach. For this reason, the construction of the fuzzy covering graph of a picture fuzzy graph is more important than any other general type fuzzy graph. It will be very helpful in the decision-making process by the help of fuzzy covering technique of picture fuzzy graphs.

Let $G^{*}=(V, E)$ be a graph. A pair $G=(A, B)$ is called a picture fuzzy graph on $G^{*}$ where $A=\left(\mu_{A}, \eta_{A}, v_{A}\right)$ is a picture fuzzy set on $V$ and $B=\left(\mu_{B}, \eta_{B}, v_{B}\right)$ is a picture fuzzy set on $E \subset V \times V$ such that for each arc $u v \in E$,

$\mu_{B}(u, v) \leq \min \left(\mu_{A}(u), \mu_{A}(v)\right), \eta_{B}(u, v) \leq \min \left(\eta_{A}(u), \eta_{A}(v)\right), v_{B}(u, v) \geq \max \left(v_{A}(u), v_{A}(v)\right)$.

Let us consider a new fuzzy graph, $H^{*}=\left(V_{2}, E_{2}\right)$ with a pair $H=(C, D)$ is also a picture fuzzy graph. Here $C=\left(\mu_{C}, \eta_{C}, \nu_{C}\right)$ is a picture fuzzy set on $V_{2}$ and $D=\left(\mu_{D}, \eta_{D}, v_{D}\right)$ is a picture fuzzy set on $E \subset V_{2} \times V_{2}$ such that for each arc $x y \in E_{2}$,

$$
\begin{aligned}
& \mu_{D}(x, y) \leq \min \left(\mu_{C}(x), \mu_{C}(y), E(x, y)\right), \eta_{D}(x, y) \leq \min \left(\eta_{C}(x), \eta_{C}(y), E(x, y)\right), v_{D}(x, y) \\
& \geq \max \left(v_{C}(x), v_{C}(y), E(x, y)\right)
\end{aligned}
$$

where, $E$ is the equality function.

Then, we have a fuzzy covering map $\tilde{f}: V_{2} \rightarrow V_{1}$ whose membership function is given by:

$$
\chi_{\tilde{f}}(x, y)=\min \left\{\left\{\mu_{A}(x) \wedge \eta_{A}(y)\right\},\left\{\mu_{A}(y) \wedge \eta_{A}(y)\right\},\left\{v_{A}(x) \vee v_{A}(y)\right\}, E(x, y)\right\} .
$$

Therefore, $H$ is a fuzzy covering graph of $G$ i.e., the fuzzy covering graph of a picture fuzzy graph is also a picture fuzzy graph. 


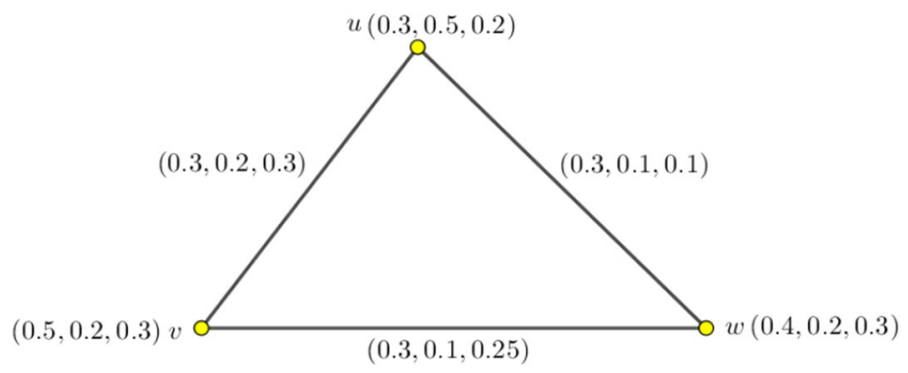

G

Fig. 10 Considered picture fuzzy graph

Example 6 Let us consider a picture fuzzy graph $G=(A, B)$ given in the Fig. 10, where $A=\left(\mu_{A}, \eta_{A}, v_{A}\right)$ is a picture fuzzy set on $V$ and $B=\left(\mu_{B}, \eta_{B}, v_{B}\right)$ is a picture fuzzy set on $E \subset V \times V$ such that for each arc $u v \in E$,

$\mu_{B}(u, v) \leq \min \left(\mu_{A}(u), \mu_{A}(v)\right), \eta_{B}(u, v) \leq \min \left(\eta_{A}(u), \eta_{A}(v)\right), v_{B}(u, v) \geq$ $\max \left(v_{A}(u), v_{A}(v)\right)$. We have to find out a fuzzy covering map together with a fuzzy covering graph of the considered picture fuzzy graph.

Assuming a fuzzy covering map $\tilde{f}: V(H) \rightarrow V(G)$ whose membership function is given by:

$\chi_{\tilde{f}}(x, y)=\min \left\{\left\{\mu_{A}(x) \wedge \eta_{A}(y)\right\},\left\{\mu_{A}(y) \wedge \eta_{A}(y)\right\},\left\{v_{A}(x) \vee v_{A}(y)\right\}, E(x, y)\right\}$.

Also, the edge-membership function for the new edges in the fuzzy covering graph is given by:

$\mu(x, y)=\left\{\begin{array}{l}\left\{\mu_{A}(x) \wedge \eta_{A}(y) \wedge E(x, y), \mu_{A}(x) \wedge \eta_{A}(y) \wedge E(x, y),\right. \\ \left.\mu_{A}(x) \vee \eta_{A}(y) \vee E(x, y)\right\} \\ \text { if } E(x, y) \in(0,0.5) \\ \left\{\mu_{A}(x) \wedge \eta_{A}(y) \wedge\{1-E(x, y)\}, \mu_{A}(x) \wedge \eta_{A}(y) \wedge\{1-E(x, y)\},\right. \\ \left.\mu_{A}(x) \vee \eta_{A}(y) \vee\{1-E(x, y)\}\right\} \\ \text { if } E(x, y) \in(0.5,1] .\end{array}\right.$

By the above consideration, we find out the fuzzy covering graph $H=(C, D)$ of the picture fuzzy graph $G=(A, B)$. The graph $H$ is also a picture fuzzy graph and given by the Fig. 11, where the yellow-coloured vertices are the existing facilities in the given picture fuzzy graph $G$ and we add the more necessary facilities among them to construct the fuzzy covering graph $H$. The new added facilities are violet-coloured vertices in $H$. 


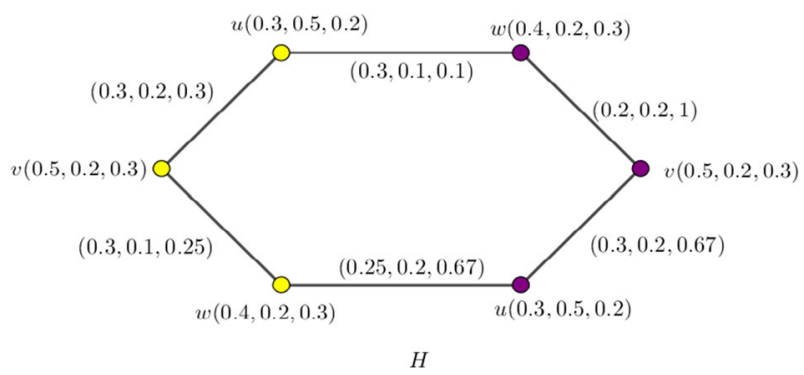

Fig. 11 Fuzzy covering graph of the picture fuzzy graph

\section{Fuzzy covering graphs of neutrosophic fuzzy graphs}

Neutrosophic fuzzy graphs are another special type of fuzzy graph which have three types of membership functions of any vertex for any result in the decision-making process. In many situations, we have to conclude a decision among three choices: truth, indeterminacy and falsity. If this particular situation needs any covering with the placements of the existing facilities in a fuzzy network within a given covering radius, then the fuzzy covering graph of neutrosophic fuzzy graphs have the most impact for maximum coverage for this purpose. In this part, the covering graph of a neutrosophic fuzzy graph is to be determined.

A neutrosophic fuzzy graph (NF-graph) with underlying set $V$ is defined to be a pair $N_{G}=(A, B)$ where,

1. The functions $T_{A}: V \rightarrow[0,1], I_{A}: V \rightarrow[0,1]$ and $F_{A}: V \rightarrow[0,1]$ denote the degree of truth-membership, degree of indeterminacy-membership and falsitymembership of the element $v_{i} \in V$, respectively, and

$$
0 \leq T_{A}\left(v_{i}\right)+I_{A}\left(v_{i}\right)+F_{A}\left(v_{i}\right) \leq 3 .
$$

2. $E \subset V \times V$ where, functions $T_{B}: V \times V \rightarrow[0,1], I_{B}: V \times V \rightarrow[0,1]$ and $F_{B}: V \times V \rightarrow[0,1]$ are defined by,

$$
\begin{aligned}
T_{B}\left(v_{i}, v_{j}\right) & \leq T_{A}\left(v_{i}\right) \cdot T_{A}\left(v_{j}\right) \\
I_{B}\left(v_{i}, v_{j}\right) & \leq I_{A}\left(v_{i}\right) \cdot I_{A}\left(v_{j}\right) \\
F_{B}\left(v_{i}, v_{j}\right) & \leq F_{A}\left(v_{i}\right) \cdot F_{A}\left(v_{j}\right)
\end{aligned}
$$

for all $v_{i}, v_{j} \in V$ where . means the ordinary multiplication; denote the degree of truthmembership, indeterminacy-membership and falsi-membership of the edge $\left(v_{i}, v_{j}\right) \in$ $E$ respectively, where

$$
0 \leq T_{B}\left(v_{i}, v_{j}\right)+I_{B}\left(v_{i}, v_{j}\right)+F_{B}\left(v_{i}, v_{j}\right) \leq 3
$$

for all $\left(v_{i}, v_{j}\right) \in E$ for $(i, j=1,2, \ldots, n)$.

We call ' $A$ ' the neutrosophic fuzzy vertex set of $V$, ' $B$ ' the neutrosophic fuzzy edge set of $E$, respectively. 


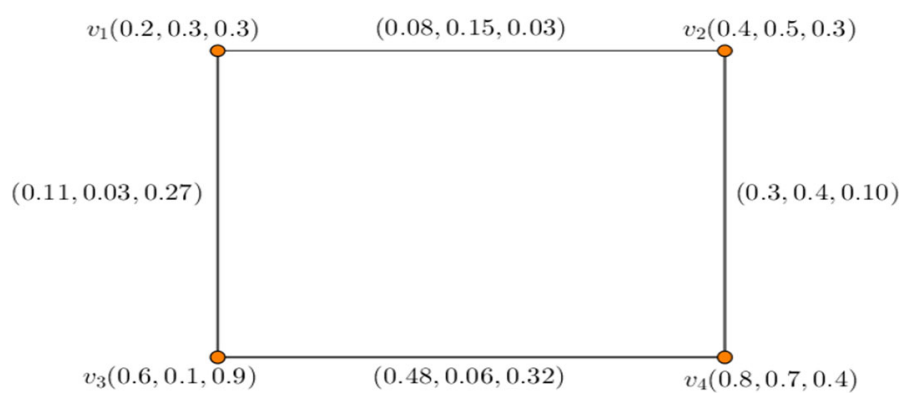

Fig. 12 Considered neutrosophic fuzzy graph $G$

Example 7 Let us consider a neutrosophic fuzzy graph $G$ given by the Fig. 12. We have to find a fuzzy covering graph of the neutrosophic fuzzy graph $G$ whose facility nodes are orange-coloured vertices.

Let, $H$ be the fuzzy covering graph of the fuzzy graph $G$. Assuming a fuzzy covering map $\tilde{f}: V(H) \rightarrow V(G)$ with the three underlying fuzzy covering maps for the three different degree membership functions of the vertices of the fuzzy graph; we have to construct the graph $H$. The membership function of the fuzzy covering map for the neutrosophic fuzzy graph is given by,

$$
\chi_{\tilde{f}}\left(v_{i}, v_{j}\right)=\min \left\{\left\{T_{A}\left(v_{i}\right) \cdot T_{A}\left(v_{j}\right)\right\} \vee\left\{F_{A}\left(v_{i}\right) \cdot F_{A}\left(v_{j}\right)\right\}, I_{A}\left(v_{i}\right) . I_{A}\left(v_{j}\right)\right\},
$$

for all $v_{i}, v_{j} \in V$.

The edge-membership function for the new edges in the fuzzy covering graph is given by,

$$
\mu\left(v_{i}, v_{j}\right)=\left\{\begin{array}{l}
\left\{T_{A}\left(v_{i}\right) \cdot T_{A}\left(v_{j}\right) \cdot E\left(v_{i}, v_{j}\right), I_{A}\left(v_{i}\right) \cdot I_{A}\left(v_{j}\right) \cdot E\left(v_{i}, v_{j}\right),\right. \\
\left.F_{A}\left(v_{i}\right) \cdot F_{A}\left(v_{j}\right) \cdot E\left(v_{i}, v_{j}\right)\right\} \\
\text { if } E\left(v_{i}, v_{j}\right) \in(0,0.5) \\
\left\{T_{A}\left(v_{i}\right) \cdot T_{A}\left(v_{j}\right) \cdot\left\{1-E\left(v_{i}, v_{j}\right)\right\}, I_{A}\left(v_{i}\right) \cdot I_{A}\left(v_{j}\right) \cdot\left\{1-E\left(v_{i}, v_{j}\right)\right\}\right. \\
\left.F_{A}\left(v_{i}\right) \cdot F_{A}\left(v_{j}\right) \cdot\left\{1-E\left(v_{i}, v_{j}\right)\right\}\right\} \\
\text { if } E(x, y) \in(0.5,1] .
\end{array}\right.
$$

for all $v_{i}, v_{j} \in V(H)$

By this consideration, we construct the graph $H$, where blue-coloured vertices are the new added facility nodes to cover the graph $G$. Here, the graph $H$ is also a neutrosophic fuzzy graph which is given by the Fig. 13 .

\section{An application}

India is one of the developing countries of the world, trying as much as possible to develop the economic situation for achieving the target of eighth SDG "Decent Work 


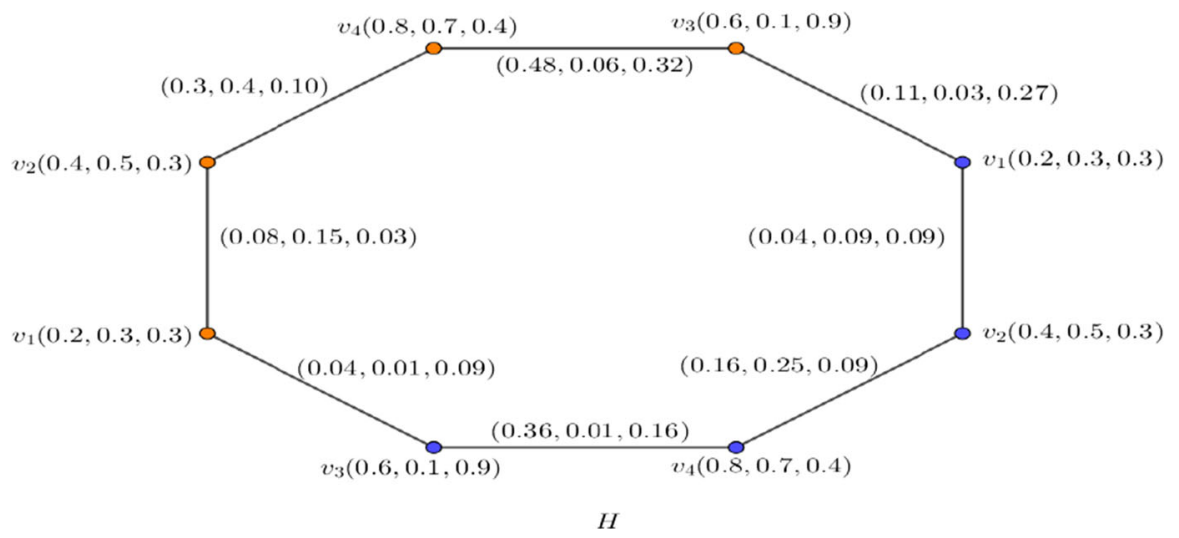

Fig. 13 Fuzzy covering graph of the neutrosophic fuzzy graph $G$

and Economic Growth". But, in the present situation, the main obstacle for fulfilling the target in eighth SDG due to a long-time lock-down process to control the pandemic COVID-19. It is very difficult for a country like India with a wide population and a developing economic status to overcome this critical situation. The economic impact of the 2020 Corona virus pandemic in India has been largely disruptive.

\subsection{SDG 8: Global context and position of India}

SDG 8 seeks to achieve higher economic productivity and job creation through diversification and innovations in technology, while at the same time protecting labour rights and promoting a safe and secure working environment. It also aims to eradicate forced labour, human trafficking and child labour. Despite gains in human development, narrowing gap of per capita income between high-and-lower-income countries and improvements in labour productivity; only limited success has been achieved globally on most of the SDG 8 targets.

With one person out of every six on the planet living in India, the country has the potential to be the engine of the global economic process. India, with a growth rate of $6.8 \%$ in 2018-2019, is recognised as one of the fastest-growing large economies in the world. With 54.3\% share of Indian GDP coming from the Services Sector, which continues to record a growth rate of 7-8 percent during each quarter of 2018-2019. India continues to register a high growth rate. The government has taken many steps to ensure further consolidation at the macro-economic level, strengthening of investment sentiments, promotion of entrepreneurship and creation of a skilled workforce. One of the key challenges in India has been the declining participation of female workers in the labour force. The seven factors of SDG 8 are:

(i) Annual growth rate of net Domestic Product (NDP) per capita $\left(f_{1}\right)$.

(ii) Ease of doing business score $\left(f_{2}\right)$.

(iii) Unemployment $\operatorname{rate}\left(f_{3}\right)$.

(iv) Labour force participation rate $\left(f_{4}\right)$. 
Table 2 Performance (Index-score) of India on indicators of SDG 8: Decent Work and Economic Growth

\begin{tabular}{|c|c|c|c|c|c|c|c|c|}
\hline States/UTs & $f_{1}$ & $f_{2}$ & $f_{3}$ & $f_{4}$ & $f_{5}$ & $f_{6}$ & $f_{7}$ & Index score \\
\hline Andhra Pradesh & 100 & 98 & 79 & 35 & 31 & 100 & 100 & 78 \\
\hline Arunachal Pradesh & 56 & 0 & 73 & 11 & 27 & 100 & 100 & 52 \\
\hline Assam & 77 & 85 & 63 & 15 & 9 & 86 & 100 & 62 \\
\hline Bihar & 100 & 82 & 67 & 0 & 0 & 100 & 100 & 64 \\
\hline Chhattisgarh & 35 & 97 & 85 & 43 & 18 & 93 & 100 & 67 \\
\hline Goa & 100 & 57 & 35 & 19 & 100 & 100 & 84 & 71 \\
\hline Gujarat & 100 & 98 & 78 & 19 & 36 & 100 & 93 & 75 \\
\hline Haryana & 80 & 98 & 61 & 12 & 54 & 100 & 92 & 71 \\
\hline Himachal Pradesh & 62 & 88 & 74 & 39 & 76 & 100 & 90 & 76 \\
\hline Jharkhand & 100 & 98 & 65 & 11 & 17 & 100 & 100 & 70 \\
\hline Karnataka & 100 & 96 & 78 & 22 & 60 & 90 & 100 & 78 \\
\hline Kerala & 63 & 45 & 47 & 13 & 57 & 100 & 100 & 61 \\
\hline Madhya Pradesh & 46 & 97 & 80 & 30 & 14 & 100 & 100 & 67 \\
\hline Maharastra & 76 & 93 & 78 & 24 & 20 & 100 & 98 & 70 \\
\hline Manipur & 0 & 0 & 46 & 16 & 6 & 17 & 100 & 27 \\
\hline Meghalaya & 91 & 0 & 93 & 40 & 29 & 100 & 100 & 65 \\
\hline Mizoram & 83 & 4 & 53 & 22 & 58 & 72 & 0 & 42 \\
\hline Nagaland & 01 & 14 & 0 & 06 & 04 & 72 & 100 & 28 \\
\hline Odisha & 63 & 92 & 67 & 16 & 24 & 48 & 100 & 59 \\
\hline Punjab & 49 & 54 & 64 & 13 & 76 & 100 & 97 & 65 \\
\hline
\end{tabular}

(v) Number of banking outlets per 1,00,000 population $\left(f_{5}\right)$.

(vi) Percentage of households with a bank account $\left(f_{6}\right)$.

(vii) Proportion of women account holders under "Pradhan Mantri Jan Dhan Yojona" $\left(f_{7}\right)$.

As per the annual report of Transforming India project 2019-2020, declared on near November, 2019; the position of India in SDG 8 based on the national index score with seven indicators are given by the Table 2 .

\subsection{Economic impact of the COVID-19 pandemic in India}

Cause: COVID-19 pandemic-induced market instability and lock-down.

Outcomes:

(i) Sharp rise in unemployment $(45 \%)$ (denoted by $a$ ).

(ii) Stress on supply chain $(53 \%)$ (denoted by $b$ ).

(iii) Decrease in government income (23.9\%) (denoted by $c$ ).

(iv) Collapse of the tourism and hospitality industry (70\%) (denoted by $d$ ).

(v) Reduced consumer activity (in Stock markets) (13.15\%) (denoted by $e$ ).

(vi) Plunge in fuel consumption and rise in LPG sales $(46 \%)$ (denoted by $f$ ).

(vii) Fall in trade with China (25\%) (denoted by $g$ ). 
Table 2 continued

\begin{tabular}{lrrrrrrrr}
\hline States/UTs & $f_{1}$ & $f_{2}$ & $f_{3}$ & $f_{4}$ & $f_{5}$ & $f_{6}$ & $f_{7}$ & Index score \\
\hline Rajasthan & 50 & 96 & 77 & 20 & 16 & 97 & 100 & 65 \\
Sikkim & 72 & 0 & 84 & 37 & 81 & 100 & 100 & 68 \\
Tamilnadu & 83 & 91 & 65 & 27 & 51 & 100 & 100 & 74 \\
Telengana & 100 & 98 & 64 & 25 & 88 & 100 & 100 & 82 \\
Tripura & 100 & 22 & 68 & 11 & 36 & 100 & 100 & 63 \\
Uttar Pradesh & 62 & 93 & 71 & 10 & 10 & 100 & 100 & 64 \\
Uttarakhand & 81 & 94 & 64 & 09 & 63 & 100 & 100 & 73 \\
West Bengal & 100 & 95 & 79 & 19 & 13 & 100 & 100 & 72 \\
Andaman \& Nicobar & 100 & 01 & 26 & 32 & 26 & 100 & 96 & 55 \\
Dadra Nagar Haveli & 0 & 22 & 98 & 46 & 41 & 100 & 73 & 63 \\
Daman \& Diu & 0 & 29 & 86 & 44 & 30 & 100 & 36 & 54 \\
Delhi & 77 & 32 & 56 & 14 & 48 & 100 & 92 & 60 \\
Jammu \& Kashmir & 54 & 33 & 75 & 25 & 36 & 0 & 97 & 46 \\
Lakshadweep & 0 & 0 & 0 & 9 & 46 & 100 & 100 & 43 \\
Puducherry & 96 & 16 & 52 & 6 & 36 & 100 & 100 & 58 \\
India & 66 & 67 & 72 & 19 & 26 & 96 & 100 & 64 \\
Target & 100 & 100 & 100 & 100 & 100 & 100 & 100 & 100 \\
\hline & & & & & & & & 54
\end{tabular}

India's growth in the fourth quarter of the fiscal year 2020 went down to $3.1 \%$ according to the Ministry of Statistics. The Chief Economic Adviser to the Government of India said that this drop is mainly due to the COVID-19 pandemic effect on the Indian economy. State Bank of India research estimates a contraction of over $40 \%$ in the GDP in Q1FY21. The contraction will not be uniform, rather it will differ according to the various parameters such as state and sector.

Unemployment rose from $6.7 \%$ on 15 th March to $26 \%$ on 19 th April and then back down to pre-lock-down levels by mid-June. During the lock-down, an estimated 14 crore (140 million) people lost employment while salaries were cut for many others. More than $45 \%$ of households across the nation have reported an income drop as compared to the previous year. The Indian Economy was expected to lose over Rs. 32,000 Crore (US $\$ 4.5$ billion) every day during the first 21 days of complete lockdown, which was declared following the corona virus outbreak. Under complete lockdown, less than a quarter of India's \$ 2.8 trillion economic movement was functional. Upto $53 \%$ of businesses in the country were projected to be significantly affected. Supply chains have been put under stress with the lock-down restrictions in place; initially, there was a lack of clarity in streamlining what an 'essential' is and what is not. A large number of farmers around the country who grow perishables also faced uncertainty.

GST is now an important term in Indian economy. GST stands for Goods and Services Tax is an indirect tax(or, consumption tax) used in India on the supply of goods and services. It is a comprehensive multistage, destination-based tax: comprehensive because it has subsumed almost all the indirect taxes except a few state taxes. 
Overall stimulus provided by Atmanirbhar Bharat Package (Rs. Cr.)

13 th to 17 th announcements

1102650

Earlier Measures incl PMGKP

192800

RBI Measures (Actual)

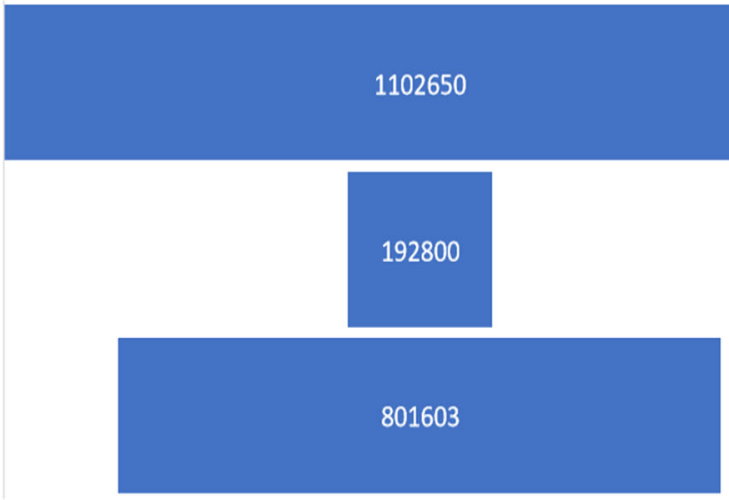

Fig. 14 The overall stimulus provided by Self-reliant India Package (Rs. Cr.)

\subsection{Self-reliant India mission}

On 12th May, 2020, the Prime Minister addressed the nation saying that the Corona virus pandemic was an opportunity for India to increase self-reliance. He proposed the "Atmanirbhar Bharat Abhiyan (Self-reliant India Mission)" economic package.

The Finance Minister stated that the aim was to 'spur growth' and 'self-reliance', adding that 'self-reliant India' does not mean cutting off from the rest of the world. The strategy of combining fiscal and monetary, liquidity measures was defended by the government. On 18th April, 2020; India changed its foreign direct investment (FDI) policy to curb 'opportunistic takeover/acquisitions of Indian Companies due to the current pandemic', according to Department for Promotion of Industry and Internal Trade. With the fall in global share prices, there is concern that China could take advantage of the situation, leading to hostile takeovers. While the new FDI policy does not restrict markets, the policy ensures that all FDI from countries that share a land border with India will now be under scrutiny of the Minister of Commerce and Industry. However, by August 2020, Chinese exports to India had fallen by $25 \%$. The overall stimulus provided by 'Atmanirbhar Bharat Package (Rs. Cr.)' is given by the Fig. 14.

\subsection{Economy recovery of India}

Arthur D. Little, an international consulting firm, has advised that India will most probably see a W-shaped recovery. Mythili Bhusnurmath writes in 'The Economic Times' that U-shaped recovery is the most likely followed by an L-shaped recovery. CRISIL chief economist says if things go well, that if the virus is contained, we can expect a V-recovery, otherwise it will end as a U-recovery.

On 2nd July, 2020; 'The Times of India' reported that a number of economic indicators such as the manufacturer's purchasing manager's index, goods movement, GST collections, electricity usage and rail freight transport showed significant improvement 
as compared to previous months under lock-down. The Reserve Bank of India had said the impact of COVID-19 is more severe than anticipated and the GDP growth during 2020-2021 is likely to remain in the negative territory. It projected some pick-up in growth impulses from the second-half (October-March) of 2020-2021 onwards.

\subsection{Fuzzy graph construction}

To analyze all the above described situations with uncertainties and impreciseness by the concept of fuzzy graphs and fuzzy covering map along with fuzzy covering graph of that fuzzy graph, we have to construct mainly two fuzzy graphs with the help of available real-life data and information. For this application, the covering radius is taken as 2 for the ease of handling the real-life fuzziness by a fuzzy graph.

(i) The first fuzzy graph is constructed to represent the performance status of India for the eighth SDG 'Decent Work and Economic Growth' with the contributions of all states/UTs of India. The national index-score for SDG 8 along with seven different factors of this SDG are considered for evaluating vertex-membership values of the fuzzy graph. For assigning the vertex membership values of the fuzzy graph, the scaling technique by dividing the index-score by 100 . Then, we get the index-score values from $[0,100]$ to a suitable membership value for vertices in $[0,1]$. Here, we consider seven vertices for seven different factors and the equality function given in the preliminaries is used to evaluate the edge-membership values of the fuzzy graph. We assume that if any factor directly or indirectly influences another factor of the SDG 8, then there is an edge between the vertices which represent these two factors. The edge-membership function is redefined for those new edges with the help of the equality function as follows:

$$
\mu(x, y)= \begin{cases}\sigma(x) \wedge \sigma(y) \wedge E(x, y) & \text { if } \quad E(x, y) \in(0,0.5) \\ \sigma(x) \wedge \sigma(y) \wedge\{1-E(x, y)\} & \text { if } \quad E(x, y) \in(0.5,1]\end{cases}
$$

This fuzzy graph is denoted by $S_{1}=\left(V_{1}, \sigma_{1}, \mu_{1}\right)$, given by the Fig. 15 .

(ii) The second fuzzy graph is developed to represent the economic impact of COVID19 pandemic in India. In real-life context, we get all the values in increasing or decreasing in all the parameters which have an important effect in the economic status of India, are all in percent value. We scaled all the data by dividing all by 100 to get the values in $[0,1]$. These scaled data are subtracted from 1 to get the positive impact of this pandemic as a complement concept. As described above, there are mainly seven areas which have a strong effect during this pandemic with long-time lock-down situations. Here, these seven factors are treated as vertices and having an influence between two vertices, then there is an edge between them. Also, the same equality function given in the preliminaries section is used to assign the edge-membership values of that fuzzy graph. The edge-membership function 


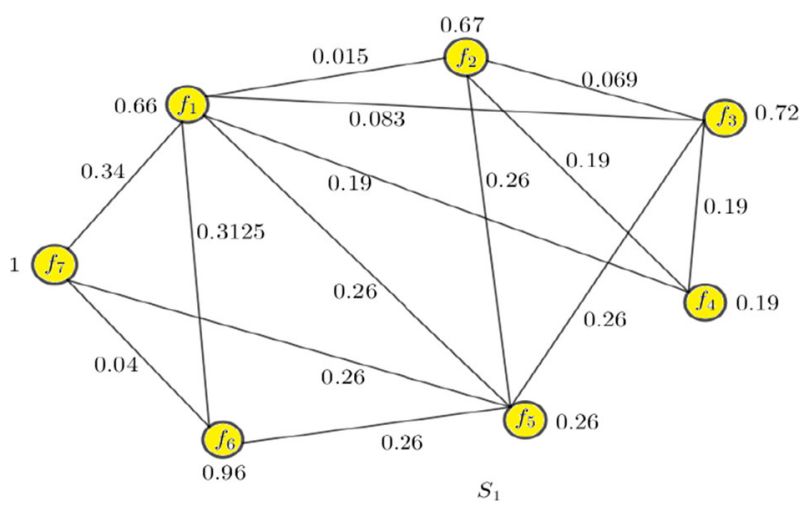

Fig. 15 Fuzzy graph representation of the performance of India for SDG 8

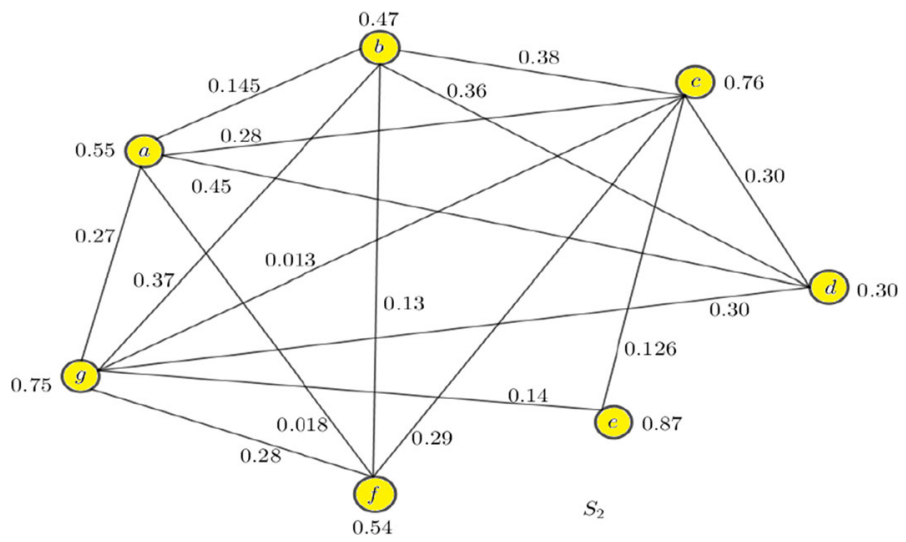

Fig. 16 Fuzzy graph representation of the impact of COVID-19 on Indian economy

is redefined for those new edges with the help of the equality function as follows:

$$
\mu(x, y)= \begin{cases}\sigma(x) \wedge \sigma(y) \wedge E(x, y) & \text { if } \quad E(x, y) \in(0,0.5) \\ \sigma(x) \wedge \sigma(y) \wedge\{1-E(x, y)\} & \text { if } \quad E(x, y) \in(0.5,1]\end{cases}
$$

This fuzzy graph is denoted by $S_{2}=\left(V_{2}, \sigma_{2}, \mu_{2}\right)$, given by the Fig. 16 .

\subsection{Covering graph for the fuzzy graph with respect to SDG 8 in India}

In this portion, the fuzzy covering graph of the fuzzy graph $S_{1}$ is to be determined by using Algorithm A1. We define a fuzzy covering map $\tilde{f}$ whose membership function, $\chi_{\tilde{f}}$ is given by $\chi_{\tilde{f}}(u, v)=\{\sigma(u) \wedge \sigma(v)\} \min \left\{\left\{E\left(u, u_{i}\right): u_{i} \in N(u)\right\} \wedge\left\{E\left(v, v_{i}\right): v_{i} \in N(v)\right\}\right\}$. 


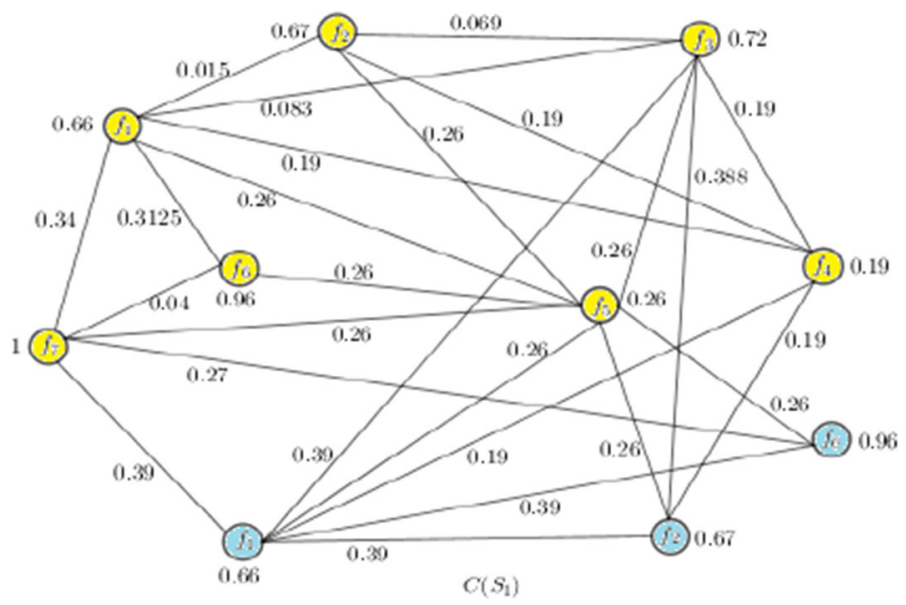

Fig. 17 Fuzzy covering graph of the fuzzy graph $S_{1}$

As in the fuzzy graph $S_{1}$, the vertices represent the effective factors for the eighth sustainable development goal "Decent Work and Economic Growth". Here $f_{1}$ i.e., annual growth rate of Net Domestic Product (NDP) per capita, $f_{2}$ i.e., ease of doing business score and $f_{6}$ i.e., percentage of households with a bank account are the most positive factors for achieving the national target for SDG 8. Therefore, to find out the fuzzy covering graph of the fuzzy graph $S_{1}$, these facility nodes i.e., $f_{1}, f_{2}$ and $f_{6}$ are included in the fuzzy covering graph to get a better coverage in the performance of India for SDG 8. Due to the inclusion of these facilities, some new edges coming out in the fuzzy covering graph. It will be noted that the membership values of the new facilities which are placed in the existing fuzzy system to get the covering graph, remain the same as in the original network. Also, the edge-membership function for the new edges in the fuzzy covering graph are determined by the following function:

$\mu(u, v)=\{\sigma(u) \wedge \sigma(v)\} \wedge\left\{\min \left\{E\left(u, u_{i}\right): u_{i} \in N(u)\right\} \vee \min \left\{E\left(v, v_{i}\right): v_{i} \in N(v)\right\}\right\}$.

Thus, the fuzzy covering graph to get a better coverage for the performance of India for SDG 8 is obtained and it is denoted by $C\left(S_{1}\right)$, given in the Fig. 17.

From Fig. 17, the new included facility nodes in $C\left(S_{1}\right)$ are coloured by different colours.

Therefore, the covering index for the fuzzy graph representing the status of India for SDG 8 is,

$$
\begin{aligned}
& (0.66+1.2005)+(0.67+0.534)+(0.72+1.38)+(0.19+0.95) \\
& +(0.26+2.08)+(0.96+0.6125)+(1+1.04)+(0.66+2.01) \\
& +(0.67+1.228)+(0.96+0.92)=18.705
\end{aligned}
$$




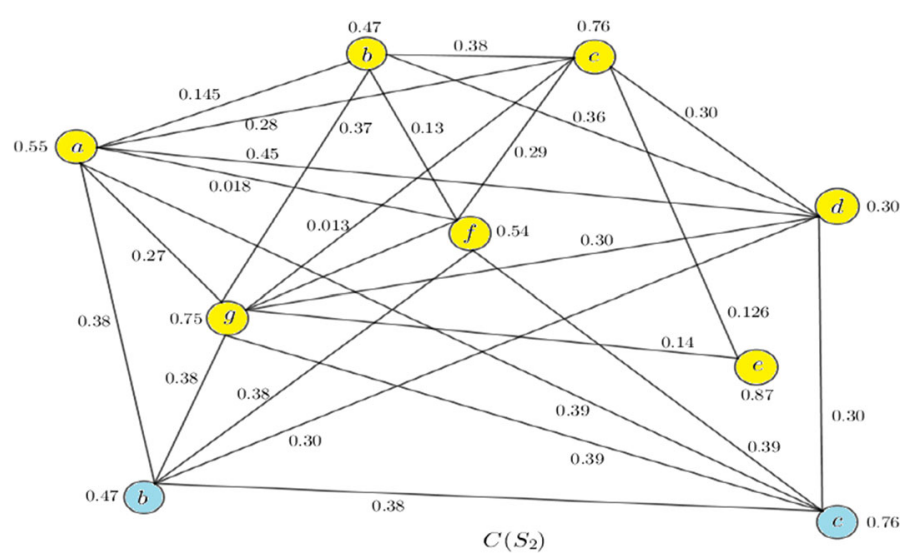

Fig. 18 Fuzzy covering graph of the fuzzy graph $S_{2}$

\subsection{Covering graph for the fuzzy graph with respect to the economic impact of COVID-19 in India}

In this part, the fuzzy covering graph of the fuzzy graph $S_{2}$ is to be determined with the help of Algorithm A1. Defining a fuzzy covering map $\tilde{f}$ which have the membership function $\chi_{\tilde{f}}$ is given by $\chi_{\tilde{f}}(u, v)=\{\sigma(u) \wedge \sigma(v)\} \min \left\{\left\{E\left(u, u_{i}\right): u_{i} \in N(u)\right\} \wedge\right.$ $\left.\left\{E\left(v, v_{i}\right): v_{i} \in N(v)\right\}\right\}$.

As said before, the impact of the current pandemic COVID-19 in the economy of the world or India is in the negative sense and has broken the backbone of the economic status of every country. Therefore, to get a positive impact of this pandemic, we subtract the negative impact scaled value from 1 and assign this value as the vertex membership value. Among the main outcomes due to this pandemic, if the two most notable outcomes $b$ i.e., stress on supply chain and $c$ i.e., decrease in government income are to be improved immediately to overcome this situation. Therefore, these two facility nodes $b$ and $c$ are to be included to determine the fuzzy covering graph of the fuzzy graph representation for this case. The vertex and edge membership functions are treated the same as the concept for constructing the fuzzy covering graph of the fuzzy graph presenting the performance status of India for SDG 8. That is, the membership values of the new facilities which are placed in the existing fuzzy system to get the covering graph, remain the same as in the original network. Also, the edge-membership function for the new edges in the fuzzy covering graph are determined by the following function:

$\mu(u, v)=\{\sigma(u) \wedge \sigma(v)\} \wedge\left\{\min \left\{E\left(u, u_{i}\right): u_{i} \in N(u)\right\} \vee \min \left\{E\left(v, v_{i}\right): v_{i} \in N(v)\right\}\right\}$

Thus, the fuzzy covering graph to get a better situation from this status due to the pandemic, is obtained and it is denoted by $C\left(S_{2}\right)$, given in the Fig. 18.

From Fig. 18, the new included facility nodes in $C\left(S_{2}\right)$ are coloured by different colour. 


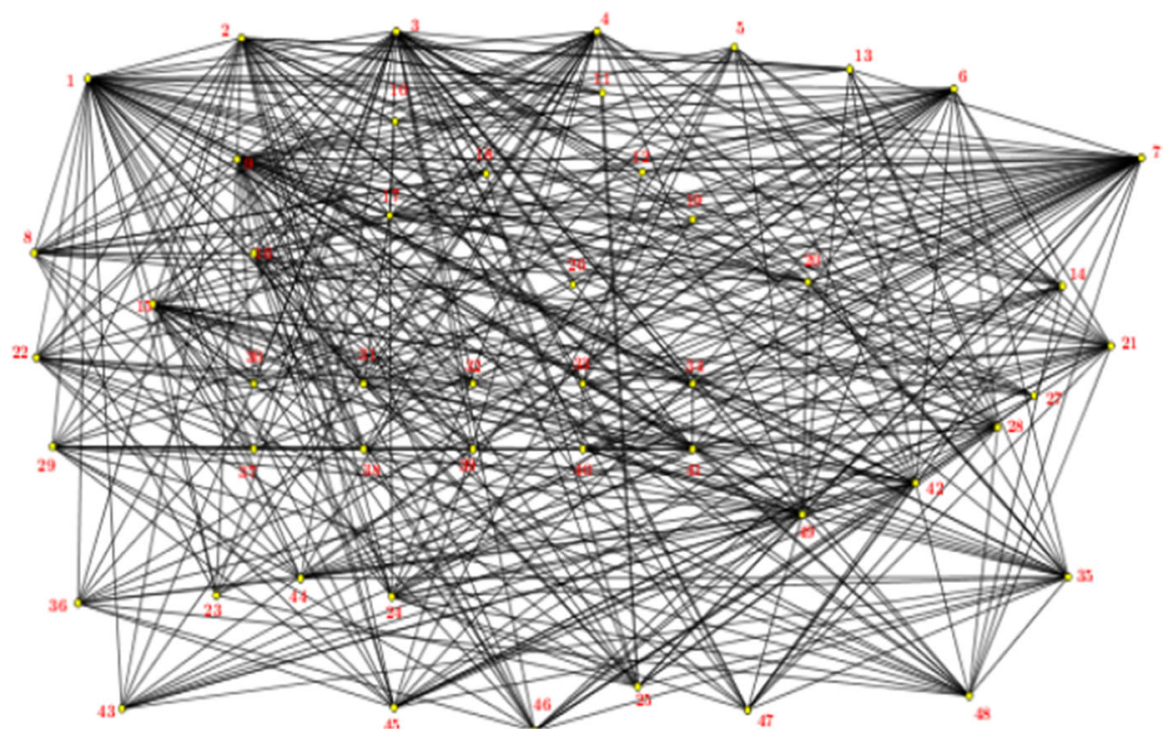

Fig. 19 Composition of two fuzzy self-covering graphs for above two cases

Therefore, the covering index for the fuzzy graph representing the status of India due to the current pandemic is,

$$
\begin{aligned}
& (0.55+1.933)+(0.47+1.385)+(0.76+1.389)+(0.30+2.01) \\
& +(0.87+0.266)+(0.54+1.488)+(0.75+2.143) \\
& \quad+(0.47+1.82)+(0.76+1.85)=19.754
\end{aligned}
$$

\subsection{Composition of two fuzzy self-covering graphs for above two cases}

Now, we find out the composition fuzzy graph of the two fuzzy self-covering graphs for performance status of India in SDG 8 and the impact of the current pandemic on Indian economy respectively. Therefore, by using the definition of composition of two fuzzy graphs, the composition of $S_{1}$ and $S_{2}$ are performed, which is given by the Fig. 19.

In the Fig. 19, for complexity of visual capacity, the vertices are denoted by $1,2,3, \ldots, 49$, also there is no naming and assignation of the vertex and edge membership values. Therefore, the detailed information is given in a tabular form by Table 3.

By the help of Table 3, the membership values of all edges present in the Fig. 19 are calculated.

Therefore, the index of the graph $S_{1} \circ S_{2}$ is given by, 72.89 .

Also, by Theorem 1 , we can conclude that $\left(S_{1} \circ S_{2}\right)_{I} \geq C_{I}\left(S_{1}\right)+C_{I}\left(S_{2}\right)$ and it is verified by the above computations. 
Table 3 Vertex membership values of the graph $\left(S_{1} \circ S_{1}\right)$

\begin{tabular}{|c|c|c|c|}
\hline Vertices & Membership value & Vertices & Membership value \\
\hline$\left(f_{1}, a\right)(1)$ & 0.55 & $\left(f_{1}, b\right)(2)$ & 0.47 \\
\hline$\left(f_{1}, c\right)(3)$ & 0.66 & $\left(f_{1}, d\right)(4)$ & 0.30 \\
\hline$\left(f_{1}, e\right)(5)$ & 0.66 & $\left(f_{1}, f\right)(6)$ & 0.54 \\
\hline$\left(f_{1}, g\right)(7)$ & 0.66 & $\left(f_{2}, g\right)(14)$ & 0.67 \\
\hline$\left(f_{2}, a\right)(8)$ & 0.55 & $\left(f_{2}, b\right)(9)$ & 0.47 \\
\hline$\left(f_{2}, c\right)(10)$ & 0.67 & $\left(f_{2}, d\right)(11)$ & 0.30 \\
\hline$\left(f_{2}, e\right)(12)$ & 0.67 & $\left(f_{2}, f\right)(13)$ & 0.54 \\
\hline$\left(f_{3}, a\right)(15)$ & 0.55 & $\left(f_{3}, b\right)(16)$ & 0.47 \\
\hline$\left(f_{3}, c\right)(17)$ & 0.72 & $\left(f_{3}, d\right)(18)$ & 0.30 \\
\hline$\left(f_{3}, e\right)(19)$ & 0.72 & $\left(f_{3}, f\right)(20)$ & 0.54 \\
\hline$\left(f_{3}, g\right)(21)$ & 0.72 & $\left(f_{4}, g\right)(28)$ & 0.19 \\
\hline$\left(f_{4}, a\right)(22)$ & 0.19 & $\left(f_{4}, b\right)(23)$ & 0.19 \\
\hline$\left(f_{4}, c\right)(24)$ & 0.19 & $\left(f_{4}, d\right)(25)$ & 0.19 \\
\hline$\left(f_{4}, e\right)(26)$ & 0.19 & $\left(f_{4}, f\right)(27)$ & 0.19 \\
\hline$\left(f_{5}, a\right)(29)$ & 0.26 & $\left(f_{5}, b\right)(30)$ & 0.26 \\
\hline$\left(f_{5}, c\right)(31)$ & 0.26 & $\left(f_{5}, d\right)(32)$ & 0.26 \\
\hline$\left(f_{5}, e\right)(33)$ & 0.26 & $\left(f_{5}, f\right)(34)$ & 0.26 \\
\hline$\left(f_{5}, g\right)(35)$ & 0.26 & $\left(f_{6}, g\right)(42)$ & 0.75 \\
\hline$\left(f_{6}, a\right)(36)$ & 0.55 & $\left(f_{6}, b\right)(37)$ & 0.47 \\
\hline$\left(f_{6}, c\right)(38)$ & 0.76 & $\left(f_{6}, d\right)(39)$ & 0.30 \\
\hline$\left(f_{6}, e\right)(40)$ & 0.87 & $\left(f_{6}, f\right)(41)$ & 0.54 \\
\hline$\left(f_{7}, a\right)(43)$ & 0.55 & $\left(f_{7}, b\right)(44)$ & 0.47 \\
\hline$\left(f_{7}, c\right)(45)$ & 0.76 & $\left(f_{7}, d\right)(46)$ & 0.30 \\
\hline$\left(f_{7}, e\right)(47)$ & 0.87 & $\left(f_{7}, f\right)(48)$ & 0.54 \\
\hline$\left(f_{7}, g\right)(49)$ & 0.75 & & \\
\hline
\end{tabular}

\subsection{Analysis of the results by the fuzzy covering graphs}

Now, from Table 3, it can be shown that the vertices 17, 19, 21, 38, 40, 45, 47, 49, 42 of the composition graph are of first category. That is, the topics presented by these vertices to make stable the Indian economy have the most important role. In the other words, the parameters combinations $\left(f_{3}, c\right),\left(f_{3}, e\right),\left(f_{3}, g\right),\left(f_{6}, c\right),\left(f_{6}, e\right),\left(f_{7}, c\right)$, $\left(f_{7}, e\right),\left(f_{7}, g\right)$ and $\left(f_{6}, g\right)$ are to be sincerely handled by the Indian government to repair the damage of this pandemic situation and to be a better performer for SDG 8 .

Now, by the real-valued data, impact of $S_{1}$ is 64, impact of $S_{2}$ is 75 respectively. By the constructed fuzzy graph data, the impact of $S_{1}$ is 18.705 , impact of $S_{2}$ is 19.754 respectively. Also, $C_{I}\left(S_{1}\right)+C_{I}\left(S_{2}\right)=38.459$ and $\left(S_{1} \circ S_{2}\right)_{I}=72.89$. With these three sets of data, a comparison is shown in the Fig. 20.

From Fig. 20, it can be concluded that the defined fuzzy covering map gives a fuzzy covering graph of the given fuzzy graphs for a better coverage by inclusion 


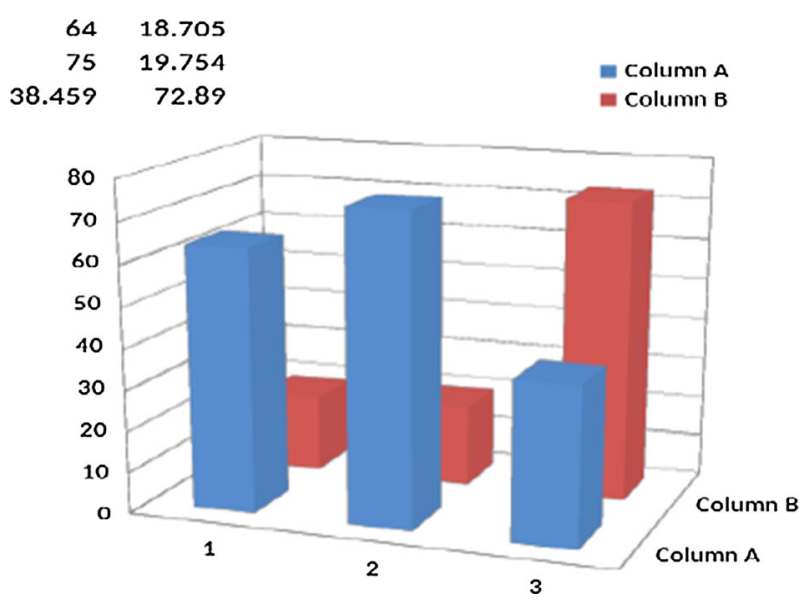

Fig. 20 Comparison analysis by three sets of data related to the application

of new facilities in the existing fuzzy network. Also, the composition of two fuzzy self-covering graphs has a greater impact than two individual fuzzy covering graphs clearly.

\subsection{Decision for this application}

From the above analysis process and discussion, for this particular application, the following are to be taken immediately:

(i) the unemployment rate $\left(f_{3}\right)$ has to be decreased in any way.

(ii) percentage of households with a bank account $\left(f_{6}\right)$ has to be increased.

(iii) women's of our society must to be empowered i.e., in another way, proportion of women account holders under PMJDY in India $\left(f_{7}\right)$ is to be increased.

(iv) the total income of the government $(c)$ must to be increased by taking all necessary steps.

(v) consumer activity throughout the country $(e)$ has to be increased by making the economy liquidity for the common people of India.

(vi) dependency in the foreign business is to be reduced $(g)$ and take more interest and importance in self-reliant India mission.

If the above are maintained, then the country will come out from this broken economic situation due to the pandemic COVID-19 and would be a better performer in fulfilling the target of eighth sustainable developmental goal 'Decent Work and Economic Growth' as a very fast developing country in the world.

\section{Conclusion}

In this paper, we introduced a new approach of covering problems of fuzzy graphs. The new definitions of fuzzy covering maps and fuzzy covering graphs of a fuzzy graph 
are introduced. The finding of fuzzy covering graphs of a fuzzy graph are discussed as another form of covering problem to locate new facility points in a existing fuzzy system. In this process, the facility location problem is solved by a new approach of fuzzy covering graphs of fuzzy graphs. Some theorems are showing the actual taste of this new concept of covering of fuzzy graphs. In the application part, a decisionmaking process based on the fuzzy covering graphs concept is illustrated. Also, a strategy making decision given for a real-life problem to overcome the pandemic situation impact on the Indian economy.

All the given new definitions and theoretical approaches will be applicable for connected, simple and undirected fuzzy graphs only. These definitions and concepts are to be modified for disconnected, directed fuzzy graphs and also other exceptional situations. In future, other types covering like the path-covering, edge-covering concepts are to be generalized by using the proposed definitions in this article. Also, the fuzzy path-covering and fuzzy edge-covering graphs of fuzzy graphs will be more relevant to model facility-location problems in new ways.

In the future work, various types of covering problems of the fuzzy graphs are to be considered to solve many real-life problems. Also, the concept of double covering of a fuzzy graph will be considered in our upcoming work. We are interested to deal with another sustainable developmental goals for a better world.

Acknowledgements Financial support of first author offered by DHESTBT (Government of West Bengal) Memo No. 353 (Sanc.)/ST/P/S\&T/16G-15/2018 is thankfully acknowledged.

Authors' contributions All the authors contribute equally in this work.

Availability of data and materials All the data are collected from 'Google'.

Code availability Not applicable.

\section{Declarations}

Conflict of interest The authors declare that they have no conflict of interest.

\section{References}

1. Al-Hawary, T., Mahamood, T., Jan, N., Ullah, K., Hussain, A.: On intuitionistic fuzzy graphs and some operations on picture fuzzy graphs. Ital. J. Pure Appl. Math. 2018, 10 (2018)

2. Akram, M.: Single-Valued Neutrosophic Graphs, Infosys Science Foundation Series in Mathematical Sciences. Springer, Berlin (2018)

3. Akram, M., Luqman, A.: A new decision-making method based on bipolar neutrosophic directed hypergraphs. J. Appl. Math. Comput. 57, 547-575 (2018)

4. Akram, M., Shahzadi, G.: Operation on single-valued NGs. J. Uncertain Syst. 11(1), 1-26 (2017)

5. Akram, M., Shahzadi, G.: Neutrosophic soft graphs with application. J. Intell. Fuzzy Syst. 32(1), 841-858 (2017)

6. Akram, M., Sitara, M.: Novel applications of single-valued neutrosophic graph structures in decisionmaking. J. Appl. Math. Comput. 56(1-2), 501-532 (2018)

7. Bhattacharya, A., Pal, M.: Vertex covering problems of fuzzy graphs and their application in CCTV installation. Neural Comput. Appl. (2020). https://doi.org/10.1007/s00521-020-05324-5 
8. Bhattacharya, A., Pal, M.: Optimization in business strategy as a part of sustainable economic growth using clique covering of fuzzy graphs. Soft Comput. (2021). https://doi.org/10.1007/s00500-02105670-Z

9. Blue, MB. Bush., Puckett, J.: Unified approach to fuzzy graph problems. Fuzzy Sets Syst. 125, 355-368 (2002)

10. Borzooei, R.A., Rashmanlou, H., Samanta, S., Pal, M.: Regularity of vague graphs. J. Intell. Fuzzy Syst. 30, 3681-3689 (2016)

11. Chang, S.S.L., Zadeh, L.: On fuzzy mappings and control. IEEE Trans. Syst. Man Cybern. 2, 30-44 (1972)

12. Chaudhry, S.S.: New heuristics for the conditional covering problem. Opsearch 30, $42-47$ (1993)

13. Dinur, I., Safra, S.: On the hardness of approximating minimum vertex cover. Ann. Math. 162(1), 1-32 (2005)

14. Dubois, D., Prade, H.: Operations on fuzzy numbers. Int. J. Syst. Sci. 9, 613-626 (1978)

15. Ghorai, G., Pal, M.: A study on m-polar fuzzy planar graphs. Int. J. Comput. Sci. Math. 7(3), 283-292 (2016)

16. Ghorai, G., Pal, M.: Faces and dual of m-polar fuzzy planar graphs. J. Intell. Fuzzy Syst. 31(3), 2043-2049 (2016)

17. Hastad, J.: Some optimal inapproximality results. J. ACM 48(4), 798-859 (2001)

18. Koczy, L.T.: Fuzzy graphs in the evaluation and optimization of networks. Fuzzy Sets Syst. 46, 307-319 (1992)

19. Mahapatra, R., Samanta, S., Pal, M., Xin, Q.: RSM: a new way of link prediction in social networks. J. Intell. Fuzzy Syst. 37, 2137-2151 (2019). https://doi.org/10.3233/JIFS-181452

20. Mahapatra, R., Samanta, S., Allahviranloo, T., Pal, M.: Radio fuzzy graphs and assignment of frequency in radio stations. Comput. Appl. Math. 38, 117 (2019). https://doi.org/10.1007/s40314-019-0888-3

21. Mahapatra, R., Samanta, S., Pal, M.: Applications of edge colouring of fuzzy graphs. Informatica 31(2), 313-330 (2020). https://doi.org/10.15388/20-INFOR403

22. Mahapatra, R., Samanta, S., Pal, M.: Generalized neutrosophic planar graphs and its application. J. Math. Comput. Appl. (2020). https://doi.org/10.1007/s12190-020-01411-X

23. Mahapatra, R., Samanta, S., Pal, M., Xin, Q.: Link prediction in social networks by neutrosophic graph. Int. J. Comput. Intell. Syst. 13(1), 1699-1713 (2020). https://doi.org/10.2991/ijcis.d.201015.002

24. Mordeson, J.N., Peng, C.S.: Operation on fuzzy graphs. Inf. Sci. 79, 159-170 (1994)

25. Naz, S., Akram, M., Smarandache, F.: Certain notions of energy in single-valued neutrosophic graphs. Axioms 7(3), 50 (2018). https://doi.org/10.3390/axioms7030050

26. Ni, Y.: Models and algorithm for stochastic minimum weight edge covering problem. In: Proceedings of the 4th International Conference on Information and Management Sciences, Yunnan, China, pp. 445-451 (2005)

27. Ni, Y.: Fuzzy minimum weight edge covering problem. Appl. Math. Model. 32, 1327-1337 (2008)

28. Pal, M., Samanta, S., Ghorai, G.: Modern trends in fuzzy graph theory. Singapore (2020). https://doi. org/10.1007/978-981-15-8803-7

29. Pramanik, T., Samanta, S., Pal, M.: Interval-valued fuzzy planar graphs. Int. J. Mach. Learn. Cybern. 7(4), 653-664 (2016)

30. Pramanik, T., Samanta, S., Sarkar, B., Pal, M.: Fuzzy $\phi$-tolerance competition graphs. Soft. Comput. 21(13), 3723-3734 (2017)

31. Radha, K., Kumaravel, N.: On edge regular fuzzy graphs. Int. J. Math. Arch. 5(9), 100-112 (2014)

32. Rashmanlou, H., Pal, M.: Isometry on interval-valued fuzzy graphs. Preprint (2014). arXiv:1405.6003

33. Rashmanlou, H., Samanta, S., Pal, M., Borzooei, R.A.: A study on bipolar fuzzy graphs. J. Intell. Fuzzy Syst. 28(2), 571-580 (2015)

34. Rosenfield, A.: Fuzzy graphs. In: Zadeh, L.A., Fu, K.S., Shimura, M. (eds.) Fuzzy Sets and Their Application, pp. 77-95. Academic Press, New York (1975)

35. Rowland, T.: Covering map. From MathWorld-A Wolfram Web Resource, Created by Eric W. Weisstein. https://www.mathworld.wolfram.com/coveringmap.html

36. Sachs, J., Schmidt Traub, G., Kroll, C., Lafortune, G., Fuller, G.: Sustainable Development Report 2019. New York: Bertelsmann Stiftung and Sustain Development Solutions Network (SDSN) (2019)

37. Sahoo, S., Pal, M.: Intuitionistic fuzzy competition graphs. J. Appl. Math. Comput. 52(1-2), 37-57 (2016)

38. Samanta, S., Akram, M., Pal, M.: m-step fuzzy competition graphs. J. Appl. Math. Comput. 47(1-2), 461-472 (2015) 
39. Samanta, S., Pal, M., Pal, A.: New concepts of fuzzy planar graph. Int. J. Adv. Res. Artif. Intell. 3(1), 52-59 (2014)

40. Samanta, S., Pramanik, T., Pal, M.: Fuzzy colouring of fuzzy graphs. Afr. Math. 27(1-2), 37-50 (2016)

41. Samanta, S., Pal, M.: Fuzzy tolerance graphs. Int. J. Latest Trends Math. 1(2), 57-67 (2011)

42. Samanta, S., Pal, M.: Fuzzy k-competition graphs and p-competition fuzzy graphs. Fuzzy Inf. Eng. 155, 1-14 (2013)

43. Samanta, S., Pal, M.: Fuzzy planar graphs. IEEE Trans. Fuzzy Syst. 23(6), 1936-1942 (2015)

44. SDG Index and Dashboards Report 2017: Global Responsibilities International, Spillovers in Achieving the Goals, Sustainable development Solutions Network, A Global Initiative for the United Nations, Bertelsmann Stiftung, May (2017), pp. 1-479

45. Talebi, A.A.: Cayley fuzzy graphs on fuzzy groups. Comput. Appl. Math. 37(4), 4611-4632 (2018)

46. Talebi, A.A., Rashmanlou, H., Sadati, S.H.: New concepts of m-polar interval valued intuitionistic fuzzy graph. TWMS J. Appl. Eng. Math. 10(3), 808-816 (2020)

47. Vinothkumar, N., Ramya, R.: Covering in operations on fuzzy graphs. Int. J. Adv. Sci. Technol. 28(16), 1127-1141 (2019)

48. Wieslaw, D., Talebi, A.A.: Operation on level graph of bipolar fuzzy graphs. Bull. Acad. Stiinte A Republic Moldova 2(8), 107-124 (2016)

49. Yager, R.R.: Knowledge-based defuzzification. Fuzzy Sets Syst. 80, 177-185 (1996)

50. Zadeh, L.A.: Fuzzy sets. Inf. Control 8, 338-353 (1965)

Publisher's Note Springer Nature remains neutral with regard to jurisdictional claims in published maps and institutional affiliations. 